\title{
Spin-wave Scattering in the Effective Lagrangian Perspective
}

\author{
Christoph P. Hofmannt \\ Department of Physics, University of California at San Diego, 9500 Gilman Drive, La Jolla, \\ California 92093
}

(May 1998)

\begin{abstract}
Nonrelativistic systems exhibiting collective magnetic behavior are analyzed in the framework of effective Lagrangians. The method, formulating the dynamics in terms of Goldstone bosons, allows to investigate the consequences of spontaneous symmetry breaking from a unified point of view. Low energy theorems concerning spin-wave scattering in ferro- and antiferromagnets are established, emphasizing the simplicity of actual calculations. The present work includes approximate symmetries and discusses the modification of the low energy structure imposed by an external magnetic and an anisotropy field, respectively. Throughout the paper, analogies between condensed matter physics and Lorentz-invariant theories are pointed out, demonstrating the universal feature of the effective Lagrangian technique.
\end{abstract}




\section{INTRODUCTION}

In the following presentation, our interest is devoted to the low energy analysis of nonrelativistic systems, which exhibit collective magnetic behavior. We adopt a unified point of view, relying on the method of effective Lagrangians, and try to understand how the symmetry, inherent in the underlying theory, manifests itself at low energies. The complex microscopic description of the systems under consideration is taken into account only through a phenomenological parametrization, which, in the effective Lagrangian, emerges in the form of a few coupling constants. Our main concern will be the question, to what extent in the low energy domain, the actual structure of quantities of physical interest is dictated by the underlying symmetry.

Nevertheless, let us first consider the Heisenberg model, which describes the magnetic systems referred to on a microscopic level. There, the exchange Hamiltonian $\mathcal{H}_{0}$,

$$
\mathcal{H}_{0}=-J \sum_{n . n .} \vec{S}_{m} \cdot \vec{S}_{n}, \quad J=\text { const. }
$$

formulates the dynamics in terms of spin operators $\vec{S}_{m}$, attached to lattice sites $m$. Note that the summation only extends over nearest neighbors and, moreover, the isotropic interaction is assumed to be the same for any two adjacent lattice sites. According to the sign of the exchange integral $J$, the above expression leads to an adequate low energy description of systems exhibiting collective magnetic behavior, both of ferro- and of antiferromagnets, respectively. In particular, the Heisenberg model is perfectly suited to study the properties of the excitations near the ground state - the spin waves or magnons.

In a more general framework, which represents the starting point of our systematic approach, these low energy excitations are interpreted as Goldstone bosons resulting from a spontaneously broken internal symmetry. Indeed, the Heisenberg Hamiltonian (1.1) is invariant under a simultaneous rotation of the spin variables, described by the symmetry group $\mathrm{G}=\mathrm{O}(3)$, whereas the ground state of a ferromagnet, e.g., breaks this symmetry spontaneously down to $\mathrm{H}=\mathrm{O}(2)$ : all the spins are aligned in one specific direction, giving rise to a nonzero spontaneous magnetization. Although the antiferromagnetic ground state does not display spontaneous magnetization, it also spontaneously breaks the symmetry. Unlike for a ferromagnet, its microscopic description is highly nontrivial - in our analysis, we assume the same internal symmetry breaking pattern to inhere in this system as well: $\mathrm{G}=\mathrm{O}(3) \rightarrow \mathrm{H}=\mathrm{O}(2)$.

Whenever a physical system exhibits spontaneous symmetry breaking and, furthermore, the corresponding Goldstone bosons represent the only low energy excitations without energy gap, we do have a very powerful means at our disposal to analyze its low energy structure: chiral perturbation theory $(\chi \mathrm{PT})_{\dot{m}}$ The method was originally developed in connection with Lorentz-invariant field theories $\mathbb{E}^{\mathrm{E}}$, admitting, in particular, a low energy analysis of the strong interaction, described by quantum chromodynamics (QCD). $\chi \mathrm{PT}$ has also proven to be very useful in the investigation of other systems where Goldstone bosons occur (see e.g.6 6 ).

In condensed matter physics, spontaneous symmetry breaking is a common phenomenon and effective field theory methods are widely used in this domain. Only recently, however, has chiral perturbation theory been extended to such nonrelativistic systems ${ }^{9}$ 12, demon- 
strating its applicability to solid state physics as well; especially the ferro- and the antiferromagnet, the systems to be examined below, may be analyzed in the framework of $\chi \mathrm{PT}$. The method is based on effective Lagrangians which exploit the symmetry properties of the underlying theory, i.e. the Heisenberg model in our case, and permits a systematic low energy expansion of quantities of physical interest in powers of inverse wavelength.

While in an anomaly free, Lorentz-invariant field theory an invariance theorem 13 guarantees that the effective Lagrangian will inherit the symmetries of the underlying model, the same statement is no longer true in the nonrelativistic domain: terms of topological nature happen to occur in the effective description, the corresponding Lagrangian being G-invariant only up to a total derivative 9, 1, 14. Especially for ferromagnets, a term connected with the Brouwer degree emerges which is not invariant under the group $\mathrm{G}=\mathrm{O}(3)$, whereas an analogous contribution is absent in the effective Lagrangian of an antiferromagnet - as we will see, the main differences in the low energy behavior of these two systems are a consequence of this striking fact.

The whole analysis concerns the properties of magnetic systems at wavelengths large compared to the intrinsic scales of the theory, i.e. to the lattice spacing $a$ - the effective theory does not resolve the lattice structure of the system, i.e. refers to the continuum limit. Clearly then, the effective Lagrangian method does not admit to discuss the physics of a solid body on a microscopic scale. Rather, we are interested in how the actual structure of several low energy phenomena encountered in magnetic systems can be interpreted as an immediate consequence of the hidden symmetry. The method has proven to be very efficient in other areas, above all in analyzing the low energy behavior of the strong interaction; in particular, the effective QCD Lagrangian allows to perform a concise derivation of certain low energy theorems concerning the pions, which represent the Goldstone bosons in this relativistic sector. The main intention of the following work is to demonstrate, that chiral perturbation theory, extended to nonrelativistic systems, is an equally powerful tool.

One principal result of the present paper will be the establishment of low energy theorems concerning the scattering amplitude of ferro- and antiferromagnetic spin waves, respectively. The straightforward effective calculation, as opposed to the complicated microscopic analysis, exhibits the efficiency of the method, which, moreover, can systematically be extended to higher orders of momentum 10 11. Likewise, it is not a complicated matter to include a weak external magnetic or an anisotropy field, respectively, into the effective machinery, in order to discuss the modifications thereby imposed on the low energy structure.

As far as the ferromagnet is concerned, our continuum approach makes contact with an important result to be found in the literature: Dyson, in his thorough microscopic analysis of a cubic ferromagnet within the Heisenberg model, calculated the scattering cross section regarding ferromagnetic spin waves more than four decades agd 15 . The fact that our result coincides with his, may be viewed as some kind of a test run for the applicability of chiral perturbation theory in the present context: the interaction among ferromagnetic spin waves is described correctly in this new framework.

In a sense to be specified below, the leading order effective Lagrangian of an antiferromagnet closely resembles the one describing QCD at lowest order. As a consequence, many results concerning chromodynamics can be adopted to antiferromagnets, the corresponding low energy phenomena manifesting themselves in analogous ways. This feature of universality offers the opportunity to discuss certain phenomena well-known in Lorentz-invariant 
theories in the different language of solid state physics and vice versa. Indeed, throughout the paper we will make quite often use of such comparisons and analogies, in order to make the material easily accessible, both to solid state physicists and to the relativistic community.

For the sake of selfconsistency of the present work, we give a brief outline of the main ideas of chiral perturbation theory and review the effective description of ferro- and antiferromagnets, leaning thereby on references 13 . 1 . In contrast to the analysis found therein, our approach tries to adopt "magnetic language", paving the way to confront our theoretical findings with the microscopic analysis.

\section{THE NONRELATIVISTIC DOMAIN}

In the following two sections, we analyze systems exhibiting collective magnetic behavior with respect to their symmetry properties, referring both to space-time and to internal transformations. Special emphasis is put into the internal symmetry $G=O(3)$ inherent in the Heisenberg model, which is spontaneously broken by the ground state of the corresponding magnetic systems. Discussing the consequences resulting from this breaking in the general framework of Goldstone's theorem, we try to work out the main differences of the low energy structure between Lorentz-invariant theories and the nonrelativistic domain.

As far as space-time symmetries are concerned, we are faced with the following situation in a continuum description of condensed matter: the object under investigation, e.g. a magnetic crystal, singles out a preferred frame of reference, the rest frame. In contrast to relativistic theories, where the vacuum is invariant under Lorentz transformations or, more generally, under the whole Poincaré group, the ground state of a solid fails to be invariant. As a consequence, the statement that the vacuum expectation value of a vector operator $A^{\mu}$ has to vanish, no longer holds: the time component $A^{0}$ may pick up an expectation value in the ground state. This observation represents an essential ingredient of the whole low energy analysis in the nonrelativistic domain, since such nonzero quantities can acquire the role of order parameters. As we soon will see, the ferromagnet is such a nonrelativistic system, where $A^{0}$ is the time component of a conserved current.

As the effective analysis refers to large wavelengths, it does not resolve the microscopic structure of a solid and the system hence appears homogeneous. Accordingly, the effective Lagrangian is invariant with respect to translations. On the other hand, the effective Lagrangian is not invariant under rotations, since the lattice structure of a solid singles out preferred directions. In the case of a cubic lattice, the anisotropy, however, only shows up at higher orders of the derivative expansion 817 In the following discussion, we assume that our magnetic systems exhibit this type of lattice structure: the underlying theory is the Heisenberg model of a cubic ferro- and antiferromagnet, respectively. Under this assumption, the leading order effective Lagrangians relating to are then invariant both under translations and under rotations.

Let us now turn to internal symmetries. In addition to the group $\mathrm{R}=\mathrm{O}(3)$, which refers to rotations in three-dimensional Euclidean space, a further symmetry group $\mathrm{O}(3)$ comes into play, associated with the isotropic exchange interaction in the Heisenberg model: $\mathrm{G}=$ $\mathrm{O}(3)$. Note that this group corresponds to internal symmetry transformations in the space of the spin variables. 
Invariance of the Heisenberg Hamiltonian $\mathcal{H}_{0}$ (1.1) with respect to the Lie group $\mathrm{G}=$ $\mathrm{O}(3)$, characterized by the generators $Q_{i}$,

$$
\left[Q_{i}, \mathcal{H}_{0}\right]=0 \text {, }
$$

gives rise to three conserved currents $J_{i}^{\mu}(x), 18$

$$
\partial_{\mu} J_{i}^{\mu}(x) \equiv \partial_{0} J_{i}^{0}(x)+\partial_{r} J_{i}^{r}(x)=0 .
$$

The generators $Q_{i}$ are space integrals over the corresponding charge densities $J_{i}^{0}(x)$,

$$
Q_{i}=\int d^{3} x J_{i}^{0}(x)
$$

obeying the commutation relations

$$
\left[Q_{i}, Q_{j}\right]=i \varepsilon_{i j k} Q_{k}
$$

The exact symmetry $\mathrm{G}=\mathrm{O}(3)$ is spontaneously broken down to $\mathrm{H}=\mathrm{O}(2)$ : whereas the Hamiltonian of the theory is invariant under the full group $\mathrm{G}$, the ground state of the system is invariant only under the subgroup $\mathrm{H}$.

In a microscopic description of a ferromagnet, e.g., this statement shows up as follows. The generators of the symmetry group are given by the sum over all spins,

$$
Q_{i}=\sum_{n} S_{n}^{i}
$$

The commutation rule

$$
\left[S_{m}^{i}, S_{n}^{j}\right]=i \delta_{m n} \varepsilon_{i j k} S_{m}^{k}
$$

insures that the Heisenberg Hamiltonian is invariant under G. The ground state of a ferromagnet, on the other hand, in which all the spins are aligned in one specific direction, let's say along the positive $3^{\text {rd }}$ axis in spin space, is invariant only under $\mathrm{H}=\mathrm{O}(2)$, represented by the single generator $Q_{3}=\sum_{n} S_{n}^{3}$.

At this point, the microscopic analysis makes contact with the continuum approach: the third component of the operator of the total spin, $\sum_{n} S_{n}^{3}$, is related to the third component of the charge density operator, $J_{3}^{0}$, by

$$
\sum_{n} S_{n}^{3}=\int d^{3} x J_{3}^{0}(x)
$$

Taking the vacuum expectation value on either side of this equation, we arrive at

$$
N S=\left\langle 0\left|J_{3}^{0}\right| 0\right\rangle V
$$

where $N$ denotes the total number of lattice sites, $S$ is the highest eigenvalue of the spin operator $S_{n}^{3}$, and $V$ is the volume of the entire crystal. Accordingly, the vacuum expectation value of the third component of the charge density operator is nonzero,

$$
\left\langle 0\left|J_{i}^{0}\right| 0\right\rangle=\delta_{i}^{3} \frac{N S}{V}=\delta_{i}^{3} \Sigma,
$$


to be identified with the spontaneous magnetization $\Sigma$. This quantity represents the most prominent order parameter in the description of a ferromagnet, its nonzero value signaling spontaneous symmetry breaking - let us elaborate this statement a bit further.

If the ground state of a ferromagnet was symmetric with respect to the whole group $\mathrm{G}=$ $\mathrm{O}(3)$, none of the operators $J_{i}^{0}(x)$, which transform in a nontrivial manner under $\mathrm{G}$, could develop a vacuum expectation value different from zero. The fact that $J_{3}^{0}(x)$ nevertheless does so, indicates, that the ground state of a ferromagnet must single out some specific direction in the internal space of the spin variables: being symmetric only with respect to the subgroup $\mathrm{H}=\mathrm{O}(2)$, the ground state does not share the full symmetry of the Hamiltonian therefore, $\left\langle 0\left|J_{3}^{0}\right| 0\right\rangle \neq 0$ may be viewed as a quantitative measure of spontaneous symmetry breaking. Generally, nonzero vacuum expectation values of local operators, which transform in a nontrivial manner under a symmetry group $\mathrm{G}$, are referred to as order parameters.

The above analysis exhibits, that the ferromagnet represents a physical system, where the most prominent order parameter is associated with the time component of a conserved current. Comparing this situation with the one in an antiferromagnet, we realize that, in this case, the spontaneous magnetization happens to vanish, $\left\langle 0\left|J_{i}^{0}\right| 0\right\rangle=0$. Although the symmetries of the ground state would have nothing against $\left\langle 0\left|J_{3}^{0}\right| 0\right\rangle$ taking on the role of an order parameter, this possibility is ruled out for dynamical reasons. As far as the antiferromagnet is concerned, the so-called staggered magnetization, $\Sigma_{s}$, turns out to be the most important order parameter - this quantity, however, is not associated with the time component of a conserved current.

\section{GOLDSTONE THEOREM}

An essential feature of the present low energy analysis is the occurrence of spontaneous symmetry breaking. The consequences of this phenomenon for the level spectrum of the corresponding systems are dictated by Goldstone's theorem.

Let us first consider its relativistic version 1921 for arbitrary Lie groups $\mathrm{G}$ and $\mathrm{H}$, associated with an internal symmetry. In the absence of gauge fields, spontaneous symmetry breaking in a Lorentz-invariant theory implies the existence of massless particles, whose number, $n_{G B}$, is determined by the dimension of the coset space $\mathrm{G} / \mathrm{H}: n_{G B}=\operatorname{dim}(\mathrm{G})-$ $\operatorname{dim}(\mathrm{H})$. The current operators $J_{a}^{\mu}$ referring to $\mathrm{G} / \mathrm{H}, a=1 \ldots n_{G B}$, couple to the vacuum, the corresponding vacuum-to-Goldstone boson matrix elements being nonzero. In QCD, e.g., the axial current, $J_{a}^{5 \mu}$, displays this property,2 2

$$
\left\langle 0\left|J_{a}^{5 \mu}\right| \pi^{b}(\vec{k})\right\rangle=i \delta_{a}^{b} k^{\mu} F .
$$

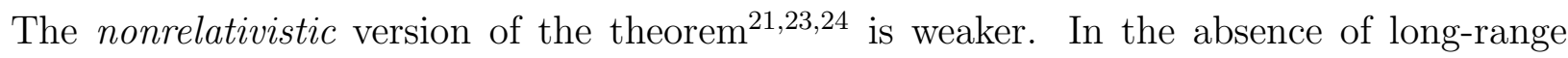
forces, spontaneous symmetry breaking in a nonrelativistic system leads to low energy excitations, whose frequency $\omega$ tends to zero for $\vec{k} \rightarrow 0$. In contrast to the relativistic version, the theorem does now neither specify the exact form of the dispersion relation at large wavelengths, nor does it determine the number of different Goldstone particles: these features of the Goldstone degrees of freedom are not fixed by symmetry considerations alone - rather, in the case of a Lorentz-noninvariant ground state, they depend on the specific properties of the corresponding nonrelativistic systems. Only the number of real Goldstone fields turns out to be universal, given again by the dimension of $\mathrm{G} / \mathrm{H}$. 
As far as the matrix elements of the operators $J_{i}^{\mu}$ between the vacuum and the Goldstone states are concerned, we find the following situation in the nonrelativistic domain: two independent coefficients have to be introduced, in order to characterize the matrix elements of the charge densities and the currents in question. Furthermore, the number of independent Goldstone states, labeled by the index $n,\left|\pi^{n}\right\rangle$, remains open. Quite generally, we may write

$$
\left\langle 0\left|J_{i}^{0}(x)\right| \pi^{n}\right\rangle=i C_{i}^{n}(\vec{k}) e^{-i k x}, \quad\left\langle 0\left|J_{i}^{r}(x)\right| \pi^{n}\right\rangle=i D_{i}^{n r}(\vec{k}) e^{-i k x} .
$$

The two quantities are related via current conservation, leading to the dispersion law 25

$$
C_{i}^{n}(\vec{k}) \omega=D_{i}^{n r}(\vec{k}) \vec{k} .
$$

Note that the exact form of the dispersion relation has not yet been specified: symmetry alone does not allow to determine the explicit $\vec{k}$-dependence of the coefficients $C_{i}^{n}(\vec{k})$ and $D_{i}^{n r}(\vec{k})$ - rather, their actual structure depends on the specific properties of the nonrelativistic systems under consideration. This is to be compared with the Lorentz-invariant situation, where the ratio of the energy to the momentum is universal, determined by the velocity of light, $\omega^{2}=c^{2} \vec{k}^{2}$ : every Goldstone boson turns out to be massless, provided that G is an exact symmetry of the Lagrangian.

From a theoretical analysis of magnetic systems, e.g. based on the Heisenberg model, as well as from the experimental side, e.g. from neutron scattering, it is well-known that the structure of the ferromagnetic dispersion relation is quite different from the antiferromagnetic one: at large wavelengths, the former takes a quadratic form, whereas the latter follows a linear law. The mechanism which leads to this pattern and, at the same time, explains the different number of independent magnon states - one for a ferromagnet, two for an antiferromagnet - is understood 26 26. Remarkably, in the framework of our effective description, the difference in the value of a single observable, the spontaneous magnetization, suffices to answer both questions: the one concerning the number of independent magnon states as well as the one referring to their dispersion lawe. We shall briefly review the chain of arguments in a later paragraph, once we have the corresponding effective Lagrangians at our disposal.

In the following analysis, the microscopic structure of the system does not play a significant role. A brief discussion of the spin-wave excitations within the Heisenberg model of a ferromagnet may be found in appendix A, which also tries to give a intuitive understanding of Goldstone's theorem in the present context.

\section{ASPECTS OF CHIRAL PERTURBATION THEORY}

Chiral perturbation theory is an efficient method to analyze the low energy structure of systems with a spontaneously broken symmetry. It is an effective theory, formulated in terms of Goldstone fields, applicable both to Lorentz-invariant theories and to the nonrelativistic domain. An essential condition for the whole framework to be consistent is the validity of three assumptions, whose significance in connection with magnetic systems, we are now going to examine in succession.

The first one supposes, that the magnons are the only excitations without energy gap. A more realistic description of a magnet faces the fact that the system admits other excitations with this property. In particular, phonons occur, representing the Goldstone bosons 
generated by the spontaneous breaking of translation invariance. We will concentrate on the magnons and disregard all other degrees of freedom - the same idealization has been used by Dyson and many others.

The exchange of magnons leads to singularities in the low energy region, particularly to poles occurring in the time-ordered correlation functions of the currents and charge densities. In the two-point function $\left\langle 0\left|T\left\{J_{i}^{r}(x) J_{k}^{s}(0)\right\}\right| 0\right\rangle$, e.g., the pole term arises from the exchange of a magnon between the two currents: the first current emits a magnon which propagates and gets absorbed by the second one. Apart from this one-magnon exchange, multimagnon exchange processes, corresponding to branch points, also occur and complicate the analysis considerably. At this moment, however, a second assumption, known as the pion pole dominance hypothesis, comes into play: one postulates that the singularities due to onemagnon exchange dominate the low energy expansion.

A third ingredient of the low energy analysis is the assumption that the residues of the pole terms, i.e. the vertices representing the interaction among the magnons, do admit an expansion in powers of inverse wavelength. Note that the correlation functions themselves, of course, cannot be expanded in this way, due to the pole terms showing up therein. This third assumption is essential in chiral perturbation theory: it allows to analyze the low energy structure of scattering amplitudes, form factors and other quantities of physical interest in a systematic manner.

In view of some applications to be presented later on, it is convenient to make use of the external field technique: one considers the response of the system to perturbations generated by suitable external fields $f_{\mu}^{i}(x)$, coupled to the currents $J_{i}^{\mu}(x)$. All the various correlation functions are collected compactly in a generating functional $\Gamma\{f\}$,

$$
e^{i \Gamma\{f\}}=\sum_{n=0}^{\infty} \frac{i^{n}}{n !} \int d^{4} x_{1} \ldots d^{4} x_{n} f_{\mu_{1}}^{i_{1}}\left(x_{1}\right) \ldots f_{\mu_{n}}^{i_{n}}\left(x_{n}\right)\left\langle 0\left|T\left\{J_{i_{1}}^{\mu_{1}}\left(x_{1}\right) \ldots J_{i_{n}}^{\mu_{n}}\left(x_{n}\right)\right\}\right| 0\right\rangle,
$$

where the external fields, $f_{\mu}^{i}(x)$, merely serve as auxiliary variables: appropriate functional derivatives of $e^{i \Gamma\{f\}}$ with respect to these quantities reproduce the correlation functions referred to above. The generating functional describes the transitions which occur when the system is perturbed by an external field, $\mathcal{H} \rightarrow \mathcal{H}-\int d^{3} x f_{\mu}^{i} J_{i}^{\mu}$, where $\mathcal{H}$ represents the Hamiltonian of the theory. In particular, $e^{i \Gamma\{f\}}$ is the probability amplitude for the system to remain in the ground state for $t \rightarrow+\infty$, if it was there at $t \rightarrow-\infty$.

Up to this point, the discussion of magnetic systems was based on a spontaneously broken internal symmetry, $\mathrm{G}=\mathrm{O}(3)$, inherent in the underlying theory, the Heisenberg model. The basic idea now in constructing an effective theory is to interpret the one-particle reducible graphs occurring in the underlying theory as tree graphs of an effective field theory, which involves magnon fields as fundamental variables: magnons are to be described by two scalar fields, denoted by $\pi^{a}(x), a=1,2$, and the pole terms generated by one-magnon exchange, e.g., arise now from magnon-field propagators.

In this language, the expansion of the vertices in powers of inverse wavelength corresponds to a derivative expansion of the effective Lagrangian. The translation of the various vertices into the corresponding terms of the effective Lagrangian is trivial: the one describing the interaction between four magnons, e.g., is represented through a term containing four magnon fields, together with a not yet specified number of space- and time-derivatives. In addition to the purely magnonic vertices, describing the interaction of magnons among themselves, 
the effective Lagrangian also contains contributions involving the external fields, which describe the transitions generated by the perturbation $f_{\mu}^{i} J_{i}^{\mu}$. The matrix element $\left\langle 0\left|f_{\mu}^{i} J_{i}^{\mu}\right| \pi^{n}\right\rangle$, e.g., which representsthe probability amplitude for the external field to excite one of the magnon states, is represented through a term linear in the fields $f_{\mu}^{i}(x), \pi^{a}(x)$. The effective Lagrangian thus merely collects the information about the various vertices occurring in the underlying theory.

In switching from the underlying theory over to the effective Lagrangian, one could think, at first sight, that the latter would simply inherit the former's internal symmetry: in connection with magnetic systems, one would therefore be inclined to construct an effective Lagrangian out of $\mathrm{O}(3)$-invariant expressions of increasing complexity - respecting, of course, the symmetry properties under space-time transformations, i.e. invariance under translations and space rotations in the present context.

This plausible way of proceeding, however, does not generally lead to correct effective Lagrangians 9,11,29. A detailed analysis of the low energy structure of nonrelativistic systems shows , that the leading order effective Lagrangian of a ferromagnet indeed is not invariant under the group $\mathrm{G}=\mathrm{O}(3)$ of the Heisenberg model. Note that this peculiarity is specific to the nonrelativistic domain and does not show up in an anomaly free, Lorentz-invariant theory - there, the effective Lagrangian can always be brought to a G-invariant form 13 .

Once the explicit effective Lagrangian at hand, chiral perturbation theory exhibits its full strength, emphasizing the simplicity of actual calculations. Moreover, the method allows to systematically take into account interactions, which explicitly break the symmetry of the underlying theory, provided that they can be treated as perturbations. As far as our magnetic systems are concerned, we will investigate the effect of an external magnetic and an anisotropy field on the structure of various low energy phenomena.

\section{LOW ENERGY BEHAVIOR OF FERRO- AND ANTIFERROMAGNETS}

We now confine our attention to the low energy properties of ferro- and antiferromagnets. First of all, the corresponding effective Lagrangians have to be written down. According to Goldstone's theorem, spontaneous symmetry breaking of the rotation group inherent in the Heisenberg model, $\mathrm{G}=\mathrm{O}(3) \rightarrow \mathrm{H}=\mathrm{O}(2)$, gives rise to two real magnon fields. It is convenient to use a covariant representation for the magnon field, replacing the two variables $\pi^{1}, \pi^{2}$ by a three-dimensional unit vector $\vec{U}=\left(U^{1}, U^{2}, U^{3}\right)$, which transforms with the vector representation of $\mathrm{G}=\mathrm{O}(3)$.

In this notation, the leading order effective Lagrangian of a ferromagnet read\$9

$$
\mathcal{L}_{\text {eff }}^{F}=\Sigma \frac{\partial_{0} U^{1} U^{2}-\partial_{0} U^{2} U^{1}}{1+U^{3}}+\Sigma f_{0}^{i} U^{i}-\frac{1}{2} F^{2} D_{r} U^{i} D_{r} U^{i}
$$

the last term being proportional to the square of the covariant derivative of $\vec{U}$,

$$
D_{r} U^{i}=\partial_{r} U^{i}+\varepsilon_{i j k} f_{r}^{j} U^{k}
$$

At leading order of the low energy expansion, the ferromagnet is thus characterized by two different low energy coupling constants, $\Sigma$ and $F$. The first term is related to a topological invariant, to the Brouwer degree of the map $\vec{U}(\pi)$. Remarkably, due to this contribution, 
which does not involve the auxiliary field $f_{0}^{i}$, the effective Lagrangian of a ferromagnet fails to be invariant under the group $\mathrm{G}=\mathrm{O}(3)$. The second term in (5.1) exhibits the same coupling constant, the spontaneous magnetization. Note that these two expressions, proportional to the order parameter, would not be permitted in Lorentz-invariant effective theories - they represent the main novelty occurring in condensed matter physics, where nonrelativistic kinematics is less restrictive than Lorentz invariance.

The ground state of an antiferromagnet, on the other hand, does not exhibit spontaneous magnetization, such that the above two contributions do not show up in the effective description of this system. The explicit expression for the leading order effective Lagrangian of an antiferromagnet is given by

$$
\mathcal{L}_{\text {eff }}^{A F}=\frac{1}{2} F_{1}^{2} D_{0} U^{i} D_{0} U^{i}-\frac{1}{2} F_{2}^{2} D_{r} U^{i} D_{r} U^{i}, \quad D_{\mu} U^{i}=\partial_{\mu} U^{i}+\varepsilon_{i j k} f_{\mu}^{j} U^{k} .
$$

As it is the case in the relativistic domain, the effective Lagrangian is invariant with respect to the symmetry group G. Since the expression (5.3) gives rise to a linear dispersion relation, it is more convenient to count energies as quantities of the same order as momenta, $\omega \propto|\vec{k}|$, rather than organizing the bookkeeping according to $\omega \propto \vec{k}^{2}$, as for the ferromagnet before. The Lagrangian also contains two effective coupling constants, $F_{1}$ and $F_{2}$. Note that the contribution involving $F_{2}^{2}$ represents the analog of the $F^{2}$-term in (5.1), whereas the first contribution, proportional to $F_{1}^{2}$, would appear in the effective Lagrangian of a ferromagnet only at subleading order.

Having the explicit leading order effective Lagrangians for ferro- and antiferromagnets at hand, we are now capable of describing the low energy behavior of these two systems.30

Let us start with the ferromagnet, whose ground state displays a nonzero spontaneous magnetization. This specific information on the system suffices to determine the corresponding leading order effective Lagrangian within our framework, which is characterized by the groups $\mathrm{G}=\mathrm{O}(3) \rightarrow \mathrm{H}=\mathrm{O}(2)$ (spontaneous symmetry breaking) and $\mathrm{R}=\mathrm{O}(3)$ (invariance under space rotations), respectively. The associated equation of motion is the Landau-Lifshitz equation, well-known in solid state physics, which describes the dynamics of ferromagnetic spin waves. Its nonrelativistic, Schrödinger-type structure - first order in time, but second order in space - for its part determines the number of independent magnon states: as only positive frequencies occur in its Fourier decomposition, a complex field is required to describe one particle - in a ferromagnet there exists only one type of spin-wave excitation. Remember that, in the nonrelativistic domain, Goldstone's theorem is too weak to make such a statement: it only predicts the number of real magnon fields, $\operatorname{dim}\{\mathrm{O}(3) / \mathrm{O}(2)\}=2$, but leaves open the number of different magnon particles. Moreover, merely claiming that the frequencies $\omega$ must tend to zero for $\vec{k} \rightarrow 0$, the theorem does not quantitatively specify the dispersion relation at large wavelengths. Its quadratic form, resulting from the effective Lagrangian (5.1),

$$
\omega(\vec{k})=\gamma \vec{k}^{2}+\mathcal{O}\left(|\vec{k}|^{4}\right), \quad \gamma \equiv \frac{F^{2}}{\Sigma}
$$

is a consequence of the Euclidean symmetry $R=O(3)$ as well as of the specific information on the ground state of the ferromagnet, concerning the nonzero value of its spontaneous magnetization. 
The effective machinery, relying on the external field technique, may now be put in operation, providing us with a derivative expansion of the correlation functions needed: the Landau-Lifshitz equation is to be solved iteratively and the respective solutions for the magnon field $U^{a}$ are to be inserted into the effective Lagrangian (5.1). At leading order, the whole information on the correlation functions is then collected compactly in the generating functional, $\left.\Gamma\{f\}\right|_{\text {tree }}=\int d^{4} x \mathcal{L}_{e f f}^{F}$, and may be obtained by taking appropriate derivatives with respect to the auxiliary fields $f$. Our interest is now devoted to the contribution proportional to $f_{0}^{a} f_{0}^{b}$, i.e. to the two-point function of the charge densities, $\left\langle 0\left|T\left\{J_{a}^{0} J_{b}^{0}\right\}\right| 0\right\rangle$, for it is this quantity which allows us to calculate the matrix element $\left\langle 0\left|J_{a}^{0}\right| \pi(\vec{k})\right\rangle$. The result is

$$
\left\langle 0\left|J_{a}^{0}\right| \pi(\vec{k})\right\rangle=\varepsilon_{a} \sqrt{\Sigma}, \quad \varepsilon_{a}=\frac{1}{\sqrt{2}}(1,-i) .
$$

Current conservation and invariance under $\mathrm{R}=\mathrm{O}(3)$ determine the corresponding spatial expression,

$$
\left\langle 0\left|J_{a}^{r}\right| \pi(\vec{k})\right\rangle=\varepsilon_{a} k^{r} \gamma \sqrt{\Sigma}=\varepsilon_{a} k^{r} F^{2} / \sqrt{\Sigma}
$$

At this stage of the effective analysis, we may look back to the general expressions (3.2) for these matrix elements. Within the effective framework, the explicit structure of the two quantities $C_{i}^{n}(\vec{k})$ and $D_{i}^{n r}(\vec{k})$ has now been determined. For ferromagnets, there exists only one polarization state, $|\pi(\vec{k})\rangle \Leftrightarrow\left|\pi^{n}\right\rangle, n=1$. In particular, the coefficient $C_{a}^{n}(\vec{k})$ does not dependent on momentum - equation (3.3) then takes the quadratic form (5.4).

As far as the antiferromagnet is concerned, quite a different low energy description emerges, because, for this system, the spontaneous magnetization happens to vanish. The corresponding equation of motion is of second order both in space and in time, its relativistic structure determining the number of independent magnon states: the Fourier decomposition contains both positive and negative frequencies, such that a single real field suffices to describe one particle. Accordingly, there exist two different types of spin-wave excitations in an antiferromagnet - as it is the case in Lorentz-invariant theories, Goldstone fields and Goldstone particles are in one-to-one correspondence. Moreover, these excitations now follow a linear dispersion relation,

$$
\omega(\vec{k})=v|\vec{k}|+\mathcal{O}\left(|\vec{k}|^{2}\right), \quad v \equiv \frac{F_{2}}{F_{1}}
$$

corresponding to a massless particle moving with velocity $v$.

The transition matrix elements of the charge densities and currents take the specific form

$$
\left\langle 0\left|J_{a}^{0}\right| \pi^{b}(\vec{k})\right\rangle=i \delta_{a}^{b}|\vec{k}| F_{2} / \sqrt{2 \omega}, \quad\left\langle 0\left|J_{a}^{r}\right| \pi^{b}(\vec{k})\right\rangle=i \delta_{a}^{b} k^{r} v F_{2} / \sqrt{2 \omega}
$$

There are now two polarization states, $\left|\pi^{a}(\vec{k})\right\rangle, a=1,2 \Leftrightarrow\left|\pi^{n}\right\rangle, n=1,2$, which are associated with the operators $J_{a}^{0}, J_{a}^{r}$, referring to the coset space G/H. Unlike for ferromagnets, the coefficient $C_{a}^{n}(\vec{k})$ does depend on momentum: equation (3.3) then leads to the linear dispersion law (5.7).

We would like to emphasize, once more, that this striking difference in the low energy behavior of ferro- and antiferromagnets, cannot be understood in terms of symmetry considerations: with respect to internal as well as space-time symmetries, the two systems are 
identical in our effective framework. Rather, the difference originates from the actual value of a single effective coupling constant, the spontaneous magnetization. The number of independent magnon states, the form of their dispersion relation, the low energy representation of scattering amplitudes, ...- the explicit appearance of all these low energy phenomena can be traced back to the different behavior of the respective ground states.

\section{EFFECTIVE COUPLING CONSTANTS}

In the nonrelativistic domain the manifold of effective coupling constants is larger than in Lorentz-invariant theories. In connection with our systems exhibiting collective magnetic behavior, the situation is the following: both for ferro- and for antiferromagnets there are two couplings to be determined at leading order: $\Sigma$ and $F$ for a ferromagnet, $F_{1}$ and $F_{2}$ for an antiferromagnet. Part of this information, as we will see, may be obtained from the dispersion relation.

In relativistic theories, Lorentz symmetry imposes a universal law, $\omega^{2}=c^{2} \vec{k}^{2}$, independent of the specific properties of the system under consideration. In the nonrelativistic domain, effective constants happen to show up in the leading order dispersion relation: for ferromagnets we obtained $\omega=\gamma \vec{k}^{2} \equiv\left(F^{2} / \Sigma\right) \vec{k}^{2}$, for antiferromagnets we got $\omega=v|\vec{k}| \equiv\left(F_{2} / F_{1}\right)|\vec{k}|$. So, on the one hand, the nonrelativistic situation is more complex: less information on the systems is available via symmetry, such that a larger number of constants has to be fixed phenomenologically. On the other hand, those combinations of low energy constants which happen to appear in the dispersion law, are comparatively easy to determine by experiment: the respective coefficients, $\gamma$ and $v$, may be obtained by scattering neutrons on a given magnetic crystal.

As far as the ferromagnet is concerned, the spontaneous magnetization is easily accessible as well - the two low energy couplings, $\Sigma$ and $F$, occurring in the leading order effective Lagrangian $\mathcal{L}_{\text {eff }}^{F}$, are thus fixed. For the antiferromagnet, which does not develop such a vacuum expectation value, the situation is more delicate. So far, we have merely been determining the ratio $v=F_{2} / F_{1}$.

Here comes the appropriate place, where we may pause for a moment and deviate into the field of the strong interaction. The point is that a close resemblance between the leading order effective Lagrangian of an antiferromagnet and the one describing QCD is observed. Since we know that the low energy sector of the strong interaction is successfully described within chiral perturbation theory, it might prove to be instructive to have a look at the specific way the low energy couplings are determined there. Maybe, reasoning by analogy, we will then be able to unravel the individual values of $F_{1}$ and $F_{2}$.

At leading order, the effective QCD Lagrangian for two massless flavors (up- and downquark mass equal to zero) involves a single coupling constant, $F_{\pi}$ (see e.g. Leutwyler Brasil),

$$
\mathcal{L}_{\text {eff }}^{Q C D}=\frac{1}{2} F_{\pi}^{2} \partial_{\mu} U^{i} \partial^{\mu} U^{i}
$$

whereas the effective Lagrangian of an antiferromagnet involves two such quantities,

$$
\mathcal{L}_{\text {eff }}^{A F}=\frac{1}{2} F_{1}^{2} \partial_{0} U^{i} \partial_{0} U^{i}-\frac{1}{2} F_{2}^{2} \partial_{r} U^{i} \partial_{r} U^{i}
$$

As it is commonly done with the velocity of light in relativistic theories, we may put the spin-wave velocity $v=F_{2} / F_{1}$ to one. In this " $\hbar=v=1$ "-system the two coupling constants 
coincide: $F_{1}=F_{2} \equiv F_{A F}$. Also, the two Lagrangians above are then the same, except for the number of fields $U^{i}$ and the actual values of the couplings $F_{\pi}$ and $F_{A F}$. In this framework, where only one low energy constant $F_{A F}$ exists, neutron scattering experiments again, of course, do not shed any light on its value - they merely fix the fundamental scale of the spin-wave velocity $v$ in the respective crystal, analogous to a determination of the velocity of light, which is then put to one.

Now, in the Standard Model of elementary particle physics, $F_{\pi}$ is related to the electroweak interaction: the constant shows up in the description of pion-decay processes and can be determined by measuring the lifetime of charged pions $-F_{\pi}$ is therefore referred to as pion decay constant. This neat way of fixing $F_{\pi}$, offered by nature, has to be regarded as a present from heaven, which, unfortunately, does not repeat itself in an analogous manner for the antiferromagnet. Later on, in connection with spin-wave scattering, we will take up the question of how to phenomenologically determine $F_{A F}$ anew.

\section{LOW ENERGY THEOREM FOR FERROMAGNETS}

Let us now turn to our original intention, namely, to study the low energy behavior of spin-wave scattering within the effective description. To begin with, consider an elastic scattering process between two identical particles,

$$
\pi\left(\vec{k}_{1}\right)+\pi\left(\vec{k}_{2}\right) \rightarrow \pi\left(\vec{k}_{3}\right)+\pi\left(\vec{k}_{4}\right) .
$$

In a nonrelativistic normalization of the one-magnon states,

$$
\left\langle\pi(\vec{k}) \mid \pi\left(\vec{k}^{\prime}\right)\right\rangle=(2 \pi)^{3} \delta^{3}\left(\vec{k}-\vec{k}^{\prime}\right),
$$

the $S$-matrix relating to is given by

$$
\begin{aligned}
S= & \left.\left\langle\pi\left(\vec{k}_{3}\right) \pi\left(\vec{k}_{4}\right) \text { out }\right| \pi\left(\vec{k}_{1}\right) \pi\left(\vec{k}_{2}\right) \text { in }\right\rangle \\
= & (2 \pi)^{6}\left\{\delta^{3}\left(\vec{k}_{1}-\vec{k}_{3}\right) \delta^{3}\left(\vec{k}_{2}-\vec{k}_{4}\right)+\delta^{3}\left(\vec{k}_{1}-\vec{k}_{4}\right) \delta^{3}\left(\vec{k}_{2}-\vec{k}_{3}\right)\right\} \\
& +i(2 \pi)^{4} \delta\left(\omega_{1}+\omega_{2}-\omega_{3}-\omega_{4}\right) \delta^{3}\left(\vec{k}_{1}+\vec{k}_{2}-\vec{k}_{3}-\vec{k}_{4}\right) T .
\end{aligned}
$$

As far as the evaluation of the $T$-matrix element is concerned, we will lean on the canonical approach, since the calculation is more readily done by means of field operators, rather than by making use of the external field technique. Within the latter framework, where one uses the equation of motion to evaluate appropriate four-point functions, the analysis of ferromagnetic spin-wave scattering, although straightforward, turns out to be tedious. The point is that, due to contributions proportional to $f_{0}^{a} U^{a}$ appearing in the effective Lagrangian, a second order iteration of the Landau-Lifshitz equation is required - accordingly, a careful bookkeeping is advised. Nonetheless, the final result coincides with the one to be obtained below: of course, it does not depend on the specific procedure used. Let us now briefly provide ourselves with the tools needed in the canonical formalism - we start with the ferromagnet and construct the corresponding magnon field operators in the interaction picture.

The Lagrangian is split up into two parts, 


$$
\mathcal{L}=\left.\mathcal{L}\right|_{f=0}+f_{\mu}^{i} J_{i}^{\mu}+\mathcal{O}\left(f^{2}\right)
$$

Making use of the effective expression for ferromagnets, $\mathcal{L} \Leftrightarrow \mathcal{L}_{\text {eff }}^{F}$ (5.1), the charge densities are identified as

$$
\left(J_{i}^{0}\right)_{e f f}=\Sigma U^{i}
$$

Recalling the transition matrix element (5.5) relating to,

$$
\left\langle 0\left|J_{a}^{0}(x)\right| \pi(\vec{k})\right\rangle=\varepsilon_{a} \sqrt{\Sigma} e^{-i k x}, \quad \varepsilon_{a}=\frac{1}{\sqrt{2}}(1,-i),
$$

we finally arrive at the representation of the magnon field operators $U^{a}$ in the interaction picture,

$$
\begin{aligned}
& U^{a}(x)=\frac{1}{\sqrt{\Sigma}} \int \frac{d^{3} k}{(2 \pi)^{3}}\left\{\varepsilon_{a} a(\vec{k}) e^{-i k x}+\varepsilon_{a}^{\star} a(\vec{k})^{\dagger} e^{i k x}\right\}, \\
& {\left[a(\vec{k}), a\left(\vec{k}^{\prime}\right)^{\dagger}\right]=(2 \pi)^{3} \delta^{3}\left(\vec{k}-\vec{k}^{\prime}\right), \quad|\pi(\vec{k})\rangle=a(\vec{k})^{\dagger}|0\rangle .}
\end{aligned}
$$

The magnon field operators to be used below, read

$$
u(x)=\sqrt{\frac{2}{\Sigma}} \int \frac{d^{3} k}{(2 \pi)^{3}} a(\vec{k}) e^{-i k x}, \quad u(x)^{\dagger}=\sqrt{\frac{2}{\Sigma}} \int \frac{d^{3} k}{(2 \pi)^{3}} a(\vec{k})^{\dagger} e^{i k x} .
$$

Note that, on the classical level, these operators correspond to the following linear combinations of the components of the magnon field $\vec{U}: u=U^{1}+i U^{2}$ and $u^{\star}=U^{1}-i U^{2}$, respectively.

Next, we determine those terms in the effective Lagrangian, which are relevant to the scattering process in question. Turning off the external fields $f_{\mu}^{i}(x)$ in the original expression $\mathcal{L}_{\text {eff }}^{F}$ (5.1) altogether, we find

$$
\left.\mathcal{L}_{e f f}^{F}\right|_{f=0}=\frac{\Sigma}{1+U^{3}} \varepsilon_{a b} \dot{U}^{a} U^{b}-\frac{1}{2} F^{2} \partial_{r} U^{i} \partial_{r} U^{i}, \quad \varepsilon_{a b}=-\varepsilon_{b a}, \quad \varepsilon_{12}=1 .
$$

Expanding the field $U^{3}$,

$$
U^{3}=\left(1-U^{a} U^{a}\right)^{1 / 2}=1-\frac{1}{2} U^{a} U^{a}-\ldots,
$$

the terms quartic in $U^{a}$, which describe the spin-wave interaction, can be read off,

$$
\mathcal{L}_{\text {int }}^{F}=\frac{1}{8} \sum \varepsilon_{a b} \dot{U}^{a} U^{b}\left(U^{c} U^{c}\right)-\frac{1}{2} F^{2}\left(U^{a} \partial_{r} U^{a}\right)\left(U^{b} \partial_{r} U^{b}\right) .
$$

Written in terms of the field operators $u$ and $u^{\dagger}$, the relevant expression is given by

$$
\mathcal{L}_{\text {int }}^{F}=\frac{1}{16} i \Sigma\left(u^{\dagger} u^{\dagger} u \dot{u}-u u u^{\dagger} \dot{u}^{\dagger}\right)-\frac{1}{8} F^{2} \partial_{r}\left(u^{\dagger} u\right) \partial_{r}\left(u^{\dagger} u\right)
$$

With the representation (7.6) of the field operators, the evaluation of the $T$-matrix element is readily done, resulting in

$$
T^{F}=\left\langle\pi\left(\vec{k}_{3}\right) \pi\left(\vec{k}_{4}\right)\left|\mathcal{L}_{\text {int }}^{F}\right| \pi\left(\vec{k}_{1}\right) \pi\left(\vec{k}_{2}\right)\right\rangle=\frac{2 \gamma}{\Sigma} \vec{k}_{1} \cdot \vec{k}_{2}
$$


For the corresponding differential cross section, we obtain (see appendix B)

$$
\frac{d \sigma^{F}}{d \Omega}=\frac{1}{32 \pi^{2} \Sigma^{2}}\left(\vec{k}_{1} \cdot \vec{k}_{2}\right)^{2} .
$$

The evaluation of the total cross section is trivial, because the $T$-matrix element (7.11), remarkably, does not depend on any angles associated with the outgoing particles. For ferromagnetic spin-wave scattering, the low energy theorem for the total cross section thus amounts to

$$
\sigma_{t o t}^{F}=\frac{1}{8 \pi \Sigma^{2}}\left(\vec{k}_{1} \cdot \vec{k}_{2}\right)^{2}
$$

This result is the same as the one Dyson derived in his microscopic theory of spin waves a long time ago 15 . Clearly, the expression obtained, although invariant under space rotations, violates Lorentz symmetry - a peculiarity, that can only occur if the ground state of the theory is Lorentz-noninvariant.

Whereas the above expression only reflects the (isotropic) S-wave part of the scattering cross section, Dyson worked out all terms to the order considered. In particular, the anisotropy of the lattice manifests itself in the scattering reaction: for each one of the three types of cubic crystals, he gets, in addition to the term (7.13), D-wave contributions. In the framework of the effective expansion, these terms only show up at next-to-leading order. Even with these additional contributions, the resulting scattering amplitude would not be the whole story: since all these expressions are real, the scattering amplitude does not satisfy the requirements imposed by unitarity. If we had included loop corrections to our tree level calculation, imaginary contributions in the scattering amplitude would then have shown up.

An analogous feature arises in quantum chromodynamics, where the next-to-leading order effective Lagrangian has been worked out already some time ago . While with the leading order effective QCD Lagrangian a concise rederivation of low energy theorems concerning the pions may be achieved, the next-to-leading order Lagrangian as well as loop graphs, originating from the leading order contribution, permit to systematically correct these theorems. In particular, imaginary terms resulting from loop graphs play a decisive role in the pion-pion scattering amplitude, which has been worked out to even higher orders 31 . As it is characteristic of the effective Lagrangian method, new effective coupling constants appear, if one extends $\chi \mathrm{PT}$ to higher orders of momentum. As far as QCD is concerned, 32 two such new couplings show up in the next-to-leading order effective Lagrangian, which are left undetermined by chiral or Lorentz symmetry, and hence have to be fixed phenomenologically. In fact, the analysis of pion-pion scattering experiments leads to a determination of these fundamental constants of chiral perturbation theory.

Unfortunately, we are not in an equally satisfactory position as far as magnetic systems are concerned: in order to experimentally detect spin-wave interactions, scattering processes are not the suitable tool - the corresponding cross section turns out to be very small (see e.g.26). As far as I know, no experiments making this quantity directly accessible, have ever been performed. Having this experimental situation in mind, it would certainly not be a very clever idea to work out the effective Lagrangian of a ferromagnet to next-toleading order, with the only intention to elaborate the analysis of spin-wave scattering further - an experimental determination of these additional effective couplings, appearing 
at higher orders of momentum, clearly cannot come from this sector. Rather, the access to some of these low energy constants will be made available by another field: applications of the method to thermodynamic quantities, such as the variation of the magnetization with temperature, may be of considerable help to carry through this program.

In any case, at leading order of the derivative expansion, the effective Lagrangian method reproduces the low energy theorem found by Dyson. Once the machinery is developed, predictions for quantities of physical interest are obtained in a concise and straightforward manner. In particular, for the total cross section to exhibit the above Lorentz-noninvariant structure, it is essential that the effective Lagrangian of a ferromagnet contains a contribution, proportional to the spontaneous magnetization, which is not invariant under the symmetry group $\mathrm{G}=\mathrm{O}(3)$ - from a methodical point of view, this is probably the main conclusion to be drawn from this section.

\section{LOW ENERGY THEOREM FOR ANTIFERROMAGNETS}

Turning now to antiferromagnetic spin-wave scattering, we are faced with a minor complication arising from the fact that there exist two independent polarization states. Accordingly, the interaction in question may generally be formulated as

$$
\pi^{a}\left(\vec{k}_{1}\right)+\pi^{b}\left(\vec{k}_{2}\right) \rightarrow \pi^{c}\left(\vec{k}_{3}\right)+\pi^{d}\left(\vec{k}_{4}\right), \quad a, \ldots, d=1,2 .
$$

Analogous to the preceding paragraph, we are going to use a nonrelativistic normalization of the one-magnon states,

$$
\left\langle\pi^{a}(\vec{k}) \mid \pi^{b}\left(\vec{k}^{\prime}\right)\right\rangle=(2 \pi)^{3} \delta^{a b} \delta^{3}\left(\vec{k}-\vec{k}^{\prime}\right),
$$

and the evaluation of the $T$-matrix element will be based on the canonical approach.

So again, the Lagrangian is split up into two parts,

$$
\mathcal{L}=\left.\mathcal{L}\right|_{f=0}+f_{\mu}^{j} J_{j}^{\mu}+\mathcal{O}\left(f^{2}\right)
$$

With the effective expression for antiferromagnets, $\mathcal{L} \Leftrightarrow \mathcal{L}_{\text {eff }}^{A F}(5.3)$, the currents are identified as

$$
\left(J_{j}^{r}\right)_{e f f}=-F_{2}^{2} \varepsilon_{i j k} \partial_{r} U^{i} U^{k} .
$$

Considering the transition matrix element (5.8) relating to,

$$
\left\langle 0\left|J_{a}^{r}(x)\right| \pi^{b}(\vec{k})\right\rangle=i \delta_{a}^{b} k^{r} v F_{2} e^{-i k x} / \sqrt{2 \omega},
$$

the magnon field operators, associated with the two polarization states, read:33

$$
\begin{aligned}
U^{a}(x) & =\frac{v}{F_{2}} \int \frac{d^{3} k}{(2 \pi)^{3} \sqrt{2 \omega}}\left\{a^{a}(\vec{k}) e^{-i k x}+a^{a}(\vec{k})^{\dagger} e^{i k x}\right\}, \\
{\left[a^{a}(\vec{k}), a^{b}\left(\vec{k}^{\prime}\right)^{\dagger}\right] } & =(2 \pi)^{3} \delta^{a b} \delta^{3}\left(\vec{k}-\vec{k}^{\prime}\right), \quad\left|\pi^{a}(\vec{k})\right\rangle=\varepsilon_{a b} a^{b}(\vec{k})^{\dagger}|0\rangle .
\end{aligned}
$$

In order to determine the relevant interaction terms in the effective Lagrangian $\mathcal{L}_{\text {eff }}^{A F}$ (5.3), we put the external fields $f_{\mu}^{i}(x)$ to zero, 


$$
\left.\mathcal{L}_{e f f}^{A F}\right|_{f=0}=\frac{1}{2} F_{1}^{2} \partial_{0} U^{i} \partial_{0} U^{i}-\frac{1}{2} F_{2}^{2} \partial_{r} U^{i} \partial_{r} U^{i},
$$

expand the variable $U^{3}$, and extract the terms quartic in $U^{a}$,

$$
\mathcal{L}_{\text {int }}^{A F}=\frac{1}{2} F_{1}^{2}\left(U^{a} \partial_{0} U^{a}\right)\left(U^{b} \partial_{0} U^{b}\right)-\frac{1}{2} F_{2}^{2}\left(U^{a} \partial_{r} U^{a}\right)\left(U^{b} \partial_{r} U^{b}\right) .
$$

We then obtain the following low energy theorem for the T-matrix element describing antiferromagnetic spin-wave scattering:

$$
\begin{gathered}
T^{A F}=\left\langle\pi^{c}\left(\vec{k}_{3}\right) \pi^{d}\left(\vec{k}_{4}\right)\left|\mathcal{L}_{\text {int }}^{A F}\right| \pi^{a}\left(\vec{k}_{1}\right) \pi^{b}\left(\vec{k}_{2}\right)\right\rangle \\
=\frac{1}{2 \sqrt{\omega_{1} \omega_{2} \omega_{3} \omega_{4}}} \frac{v^{4}}{F_{2}^{2}}\left\{\delta^{a b} \delta^{c d}\left(\left|\vec{k}_{1}\right|\left|\vec{k}_{2}\right|-\vec{k}_{1} \cdot \vec{k}_{2}\right)-\delta^{a c} \delta^{b d}\left(\left|\vec{k}_{1}\right|\left|\vec{k}_{3}\right|-\vec{k}_{1} \cdot \vec{k}_{3}\right)\right. \\
\left.\quad-\delta^{a d} \delta^{b c}\left(\left|\vec{k}_{1}\right|\left|\vec{k}_{4}\right|-\vec{k}_{1} \cdot \vec{k}_{4}\right)\right\} .
\end{gathered}
$$

As far as actual measurements of the scattering cross section relating to are concerned, we are in an equally unsatisfactory position as that of the ferromagnet before. This experimental dead end is indeed highly unwelcome, since we have not yet been able to determine the individual values of the two low energy constants $F_{1}$ and $F_{2}$, occurring in the leading order effective Lagrangian of the antiferromagnet. From the dispersion law we merely know their ratio, $v=F_{2} / F_{1}$. Note that the constant $F_{2}$ shows up separately in formula (8.6). In principle then, a measurement of the corresponding cross section would offer the possibility to fix this constant and hence allow to extract the other coupling $F_{1}$ from experimental data.

Turning now to the theoretical side, the literature on antiferromagnetic spin-wave scattering appears to be rather scarce. Unlike for the ferromagnet, where, after Dyson's monumental work, a whole lot of publications on the subject showed up (some of them trying to simplify his calculations and rederive his results, see e.g.34), only a few references dealing with the analogous problem in antiferromagnets seem to be available. References 35 rely on a microscopic description of the antiferromagnet, while reference 38 approaches the subject on the basis of a phenomenological theory. However, these authors rather direct their attention to other aspects of the spin-wave interaction. Moreover, the paper of Brooks Harris 37 appears to be the only one which is in agreement with the energy-momentum dependence of the scattering amplitude (8.6) obtained above.

From a methodical point of view, it is instructive to compare the result regarding antiferromagnetic spin-wave scattering with what is known about the analogous item in QCD: pion-pion scattering. There, at leading order of the effective expansion, the $T$-matrix element in question takes the Lorentz-invariant form 39 (see e.g.

$$
T^{Q C D}=\frac{1}{F_{\pi}^{2}}\left\{\delta^{a b} \delta^{c d} s+\delta^{a c} \delta^{b d} t+\delta^{a d} \delta^{b c} u\right\},
$$

where $s, t, u$ denote the Mandelstam variables,

$$
s=\left(k_{1}+k_{2}\right)^{2}, \quad t=\left(k_{1}-k_{3}\right)^{2}, \quad u=\left(k_{1}-k_{4}\right)^{2} .
$$

The indices $a, \ldots, d$ in (8.7), labeling the three different isospin states, are analogous to the ones needed to denote the two independent polarization states of antiferromagnetic magnons in (8.6). Comparing these two amplitudes, we see that the energy-momentum dependence 
is the same: the respective terms in (8.6) may be viewed as scalar products of momentum four vectors. As a matter of fact, the analogy between the two expressions is even more pronounced. If, just for the moment, a relativistic normalization of the one-magnon states is used and the spin-wave velocity $v$ is put to one, " $\hbar=v=1 " \rightarrow F_{1}=F_{2} \equiv F_{A F}$, then they formally coincide: apart from the number of independent Goldstone states and the actual values of the constants $F_{\pi}$ and $F_{A F}$, the two formulas are identical.

Clearly, this finding does not come about unexpectedly. The similarity between the effective Lagrangian of an antiferromagnet and the one of QCD is transferred to the scattering amplitudes: they exhibit analogous low energy representations. In fact, the above example may serve as a nice illustration of a characteristic feature of the effective Lagrangian technique - universality. Let us close this paragraph with some remarks on the subject.

In the construction of the effective Lagrangian, the specific properties of the underlying theory do not matter: they merely affect the numerical values of the coupling constants appearing in $\mathcal{L}_{\text {eff }}$. The only relevant information is the structure of the two groups $\mathrm{G}$ and $\mathrm{H}$, associated with the exact symmetry of the underlying theory - the low energy description turns out to be universal. Now, QCD with two massless flavors displays an exact $\mathrm{SU}(2)_{R} \times \mathrm{SU}(2)_{L}$ symmetry which is spontaneously broken to $\mathrm{SU}(2)_{V}$; these two groups are locally isomorphic to $\mathrm{G}=\mathrm{O}(4)$ and $\mathrm{H}=\mathrm{O}(3)$, respectively. Hence, the analogy to the $\{\mathrm{O}(3) \rightarrow \mathrm{O}(2)\}$-antiferromagnet, considered in this paper, is almost perfect: except for the magnitude of the constants $F_{A F}$ and $F_{\pi}$, the two effective Lagrangians also differ in the number of Goldstone particles.

Since ferro- and antiferromagnets, in our approach, are undistinguishable from the point of view of symmetry, these two nonrelativistic systems should actually provide us with a perfect illustration of the universality concept. It so happens, however, that, for the latter system, one of the low energy constants, the spontaneous magnetization, turns out to be zero. As a consequence, the effective Lagrangians relating to are apparently different, although, in either case, their construction is based on the symmetry groups $\mathrm{G}=\mathrm{O}(3)$ and $\mathrm{H}=$ $\mathrm{O}(2)$ inherent in the Heisenberg model. Note that, nonetheless, the concept of universality applies - the specific properties of an antiferromagnet, however, manifest themselves in a rather drastic way.

This striking difference in the structure of these two Lagrangians on the effective level is quite remarkable, because, in the underlying theory, the respective Hamiltonians only differ in the sign of the exchange integral $J$. A contragredient behavior, now really illuminating the concept of universality, concerns the antiferromagnet and the strong interaction: although the underlying theories, the Heisenberg model and QCD, respectively, are completely different, the corresponding effective Lagrangians are almost the same.

\section{EXTERNAL MAGNETIC FIELD}

Up to now, the analysis of spontaneous symmetry breaking was related to exact symmetries: it was assumed that the underlying theory is invariant with respect to an internal symmetry group G. In what follows in the remaining part of this presentation, we will let aside this idealization and direct our attention to approximate symmetries. The low energy phenomena considered so far will be studied in this extended framework and their modification, imposed by explicit symmetry breaking, will be discussed. 10 
As a first example of explicit symmetry breaking, let us work out the effect of an external magnetic field on the low energy behavior of ferro- and antiferromagnets. On a microscopic level, the interaction between a constant magnetic field $\vec{H}$ and the spin degrees of freedom is taken into account through the Zeeman term. In the corresponding extension of the Heisenberg model, 11

$$
\mathcal{H}=\mathcal{H}_{0}-\mu \sum_{n} \vec{S}_{n} \cdot \vec{H},
$$

the magnetic field is coupled to the vector of the total spin. Whereas $\mathcal{H}_{0}$ is invariant under a simultaneous rotation of the spin variables, the second term explicitly breaks the symmetry with respect to the group $\mathrm{G}=\mathrm{O}(3)$. In the effective Lagrangian framework, the interaction with a magnetic field corresponds to the term $\int d^{3} x f_{0}^{i} J_{i}^{0}$ : the operator of the total spin, $\sum_{n} \vec{S}_{n}$, is to be identified with $\int d^{3} x \vec{J}^{0}$, while the magnetic field $\vec{H}$, playing the role of a symmetry breaking parameter, is related to the time components of the auxiliary field, $f_{0}^{i}=\mu H^{i}$.

Independently of whether the effective description refers to a ferro- or an antiferromagnet, an external magnetic field is taken into account through the quantities $f_{0}^{i}(x)$. Apart from the identification $f_{0}^{i}=\mu H^{i}$, nothing further has to be done - the effective machinery developed earlier applies as it stands. However, in order to obtain the change in low energy structure induced by the magnetic field, the effective expansion is to be performed around the nonzero, constant value of $\mu H^{i}$ appearing in the underlying theory, i.e. in the extended Heisenberg model (9.1).

As far as the ferromagnet is concerned, the magnetic field $\vec{H}=(0,0, H), H>0$, couples to the order parameter: it enters the leading order effective Lagrangian (5.1) through a term proportional to the spontaneous magnetization,

$$
\mathcal{L}_{e f f}^{F}(\vec{H})=\Sigma \frac{\varepsilon_{a b} \partial_{0} U^{a} U^{b}}{1+U^{3}}+\Sigma \mu H^{i} U^{i}-\frac{1}{2} F^{2} \partial_{r} U^{i} \partial_{r} U^{i} .
$$

Expanding $U^{3}=\left(1-U^{a} U^{a}\right)^{1 / 2}$ in powers of the two components $U^{a}, a=1,2$, the term in question gives rise to the following contributions:

$$
\Sigma \mu H^{i} U^{i}=\Sigma \mu H\left(1-\frac{1}{2} U^{a} U^{a}-\frac{1}{8} U^{a} U^{a} U^{b} U^{b}-\ldots\right) .
$$

The linearized equation of motion shows that, in the presence of an external magnetic field, the dispersion law of ferromagnetic spin waves keeps its quadratic structure, the corresponding coefficient $\gamma$ being unchanged. The energy of the single spin-wave branch, $u=U^{1}+i U^{2}$, is merely shifted by a constant amount, proportional to the symmetry breaking parameter,

$$
\omega=\gamma \vec{k}^{2}+\mu H .
$$

Much like an approximate chiral symmetry provides the pions with a mass, an approximate symmetry with respect to internal rotations, $\mathrm{G}=\mathrm{O}(3)$, causes an energy gap in the spinwave spectrum of a ferromagnet, $\Delta \omega=\mu H$. Note that the spontaneous magnetization drops out in this expression: the energy gap is determined by the measure of explicit symmetry breaking alone.

Due to the term quartic in the magnon variables in (9.2), the scattering amplitude of ferromagnetic spin waves seems to experience a modification by the magnetic field as well. 
However, the resulting extra term in the T-matrix element is canceled by the contribution originating from the unperturbed Lagrangian (7.10), evaluated with the dispersion relation (9.3) - hence, to the order considered, the interaction in question is not affected by a magnetic field.

As far as the antiferromagnet is concerned, an external magnetic field does not manifest itself in an analogous manner in the low energy expansion: terms involving the spontaneous magnetization do not occur in the effective Lagrangian (5.3). Rather, the magnetic field appears in the time component of the covariant derivative of $\vec{U}$,

$$
\begin{array}{r}
\mathcal{L}_{\text {eff }}^{A F}(\vec{H})=\frac{1}{2} F_{1}^{2} D_{0} U^{i} D_{0} U^{i}-\frac{1}{2} F_{2}^{2} \partial_{r} U^{i} \partial_{r} U^{i}, \\
D_{0} U^{i}=\partial_{0} U^{i}+\varepsilon_{i j k} \mu H^{j} U^{k} .
\end{array}
$$

Concentrating on those contributions which involve the magnetic field, the expansion yields

$$
F_{1}^{2} \mu H\left\{-\varepsilon_{a b} \partial_{0} U^{a} U^{b}+\frac{1}{2} \mu H U^{a} U^{a}\right\}
$$

The linearized equation of motion leads to the dispersion relation

$$
\omega_{ \pm}=v|\vec{k}| \pm \mu H
$$

In the presence of $\vec{H}$, the dispersion law of antiferromagnetic spin waves keeps its linear structure - as for a ferromagnet before, it is merely shifted by a constant amount, proportional to the symmetry breaking field. Note that the two independent spin-wave branches, $u=U^{1}+i U^{2}$ and $u^{\star}=U^{1}-i U^{2}$, respectively, are affected in distinct ways: the magnetic field lifts their degeneracy by splitting them up symmetrically. Remarkably, the magnetic field does not give rise to a "mass term": in the case of a relativistic dispersion relation, as we see it here with the antiferromagnet, such a term would show up under a square root,

$$
\omega=\sqrt{v^{2} \vec{k}^{2}+v^{4} M_{G B}^{2}}
$$

Finally, let us consider the effect of an external magnetic field on antiferromagnetic spinwave scattering. Remarkably, the expansion (9.4) does not contain any terms quartic in the magnon fields. Now, in order to evaluate the T-matrix element referring to the unperturbed effective Lagrangian (8.5) with the dispersion relation (9.5), we have to choose the representation of the two polarization states accordingly: $\left|\pi^{+}\right\rangle\left(\left|\pi^{-}\right\rangle\right)$corresponds to the spin-wave branch $u=U^{1}+i U^{2}\left(u^{\star}=U^{1}-i U^{2}\right)$, which experiences a positive (negative) shift by $\vec{H}$. The calculation shows that the respective T-matrix elements do not receive additional terms from the magnetic field.

Take for example the reaction $\pi^{+}\left(\vec{k}_{1}\right)+\pi^{-}\left(\vec{k}_{2}\right) \rightarrow \pi^{-}\left(\vec{k}_{3}\right)+\pi^{+}\left(\vec{k}_{4}\right)$, which yields

$$
\begin{aligned}
T^{A F}(\vec{H}) & =\left\langle\pi^{-}\left(\vec{k}_{3}\right) \pi^{+}\left(\vec{k}_{4}\right)\left|\mathcal{L}_{i n t}^{A F}\right| \pi^{+}\left(\vec{k}_{1}\right) \pi^{-}\left(\vec{k}_{2}\right)\right\rangle \\
= & \frac{1}{4 \sqrt{\omega_{1} \omega_{2} \omega_{3} \omega_{4}}} \frac{v^{2}}{F_{2}^{2}}\left\{\left(\omega_{1}+\omega_{3}\right)^{2}-v^{2}\left(\vec{k}_{1}+\vec{k}_{3}\right)^{2}\right\} .
\end{aligned}
$$

The magnetic field drops out in the sum $\omega_{1}+\omega_{3}$ - it only appears in the denominator of the scattering amplitude, which exhibits the dispersion relation (9.5). 


\section{ANISOTROPY FIELD}

While the preceding section was devoted to a single symmetry breaking parameter, an external magnetic field, we would now like to discuss the question of explicit symmetry breaking from a general point of view. In the case of an approximate symmetry, the Lagrangian of the underlying theory contains contributions, which explicitly break the internal symmetry associated with the group G,

$$
\mathcal{L}=\mathcal{L}_{0}+m_{\alpha} O^{\alpha}
$$

Whereas the first term represents the invariant part, the operators $O^{\alpha}$ transform nontrivially under the symmetry group G. The constants $m_{\alpha}$, for their part, play the role of symmetry breaking parameters.

In this perspective, the interaction of an external magnetic field with the spin degrees of freedom represents a special case: the operators $O^{\alpha}$ are to be identified with the charge densities $J_{i}^{0}$, and are thus related to the generators $Q_{i}$ of the group G. Hence, in the effective description, the symmetry breaking parameters $m_{\alpha}$ of the underlying theory are to be taken into account through the time components of the external field $f_{0}^{i}(x)$ - in connection with explicit symmetry breaking, these auxiliary fields, as we have seen before, acquire physical significance.

If the operators $O^{\alpha}$ are not related to the generators of the group $\mathrm{G}$, then the effective Lagrangian has to be enlarged, including additional contributions which take into account the approximate character of the spontaneously broken symmetry. It is convenient to extend the effective machinery accordingly, treating the corresponding symmetry breaking parameters $m_{\alpha}$ also as external fields, $m_{\alpha}(x)$, on the same footing as the vector fields, $f_{\mu}^{i}(x)$, associated with the currents and charge densities. The generating functional then contains two arguments, $\Gamma=\Gamma\{f, m\}$. Correlation functions of the novel operators $O^{\alpha}$ may be obtained the same way as those involving the currents and charge densities. The only modification brought about by the fields $m_{\alpha}(x)$ is that the low energy expansion of the functional $\Gamma\{f, m\}$ now amounts to a double series - in an expansion in powers of the external fields $f_{\mu}^{i}(x)$, as well as in an expansion in powers of the quantities $m_{\alpha}(x)$, associated with the novel operators $O^{\alpha}$. As a prototype of this more general way of symmetry breaking, we mention the quark mass term, $\bar{q} \mathcal{M} q$, of the QCD Lagrangian: if the quark masses, playing the role of symmetry breaking parameters, are taken at their physical, nonzero values, chiral symmetry is explicitly broken. An application in connection with nonrelativistic systems will be given below.

From a methodical point of view, the following observation related to order parameters is of interest. Concerning the nature of these quantities, nonrelativistic kinematics, as we have seen, is less restrictive: in the case of a Lorentz-noninvariant ground state, the time components of conserved currents may develop such nonzero vacuum expectation values. Now, this type of order parameter, $\left\langle 0\left|J_{i}^{0}\right| 0\right\rangle$, already shows up in the effective theory, if the underlying theory is symmetric - the ferromagnet represents such a system, where the spontaneous magnetization embodies this possibility. Similarly, the vacuum expectation values of the more general operators $O^{\alpha}$, which are not related to the generators of the group $\mathrm{G}$, also represent order parameters, which, for their part, may occur both in Lorentz-invariant theories and in the nonrelativistic domain. However, this type of order parameter only shows 
up in the effective theory, if the symmetry of the underlying theory is approximate - on the effective level, the quantities $\left\langle 0\left|O^{\alpha}\right| 0\right\rangle$ then appear in association with the fields $m_{\alpha}(x)$. As an illustration, referring to the relativistic domain, we quote $\mathrm{QCD}$, where the quark condensate, $\langle 0|\bar{q} q| 0\rangle$, represents the order parameter in question. Likewise, the staggered magnetization $\Sigma_{s}$ of an antiferromagnet may serve as an example of an order parameter relevant to condensed matter physics, which belongs to this more general class.

In what follows, we are going to consider a further extension of the Heisenberg model,

$$
\mathcal{H}=\mathcal{H}_{0}-\mu \sum_{n} \vec{S}_{n} \cdot \vec{H}-\mu \sum_{n}(-1)^{n} \vec{S}_{n} \cdot \vec{h}
$$

which illustrates the concept of explicit symmetry breaking exposed above. The two fields, $\vec{H}$ and $\vec{h}$, are assumed to be weak, such that the respective interaction terms involving the spin degrees of freedom, may be considered as a perturbation of the isotropic Heisenberg Hamiltonian $\mathcal{H}_{0}$. In the Zeeman term, the sum over the spin operators is associated with the spontaneous magnetization, $\vec{\Sigma} \propto\left\langle 0\left|\sum_{n} \vec{S}_{n}\right| 0\right\rangle$, while the vacuum expectation value of the second sum is related to the staggered magnetization, $\vec{\Sigma}_{s} \propto\left\langle 0\left|\sum_{n}(-1)^{n} \vec{S}_{n}\right| 0\right\rangle$.

The field $\vec{h}$ in (10.2) corresponds to those symmetry breaking parameters $m_{\alpha}$, which are not associated with the generators of the group G. Since the quantity $\vec{h}$, much like a magnetic field, transforms with the vector representation of $G=O(3)$, the corresponding additional contribution in the leading order effective Lagrangian exhibits the same structure as the effective representation of the Zeeman term (9.2),

$$
\mathcal{L}_{\text {eff }}(\vec{h})=\Sigma_{s} \mu h^{i} U^{i} .
$$

The staggered magnetization, $\Sigma_{s}$, enters the leading order Lagrangian in the form of a new coupling constant, whose value has yet to be determined.

As we have seen earlier, a term proportional to the spontaneous magnetization does not appear in the effective Lagrangian of the antiferromagnet. For ferromagnets, however, the contribution (9.2) is substantial: it describes the modification of the low energy structure imposed by a magnetic field. On the other hand, the term (10.3), which is proportional to the staggered magnetization, does not show up in the effective Lagrangian of the ferromagnet considered in this paper: in the case of identical spins at each lattice site, we have $\Sigma_{s}=0$. However, for antiferromagnets, it is the staggered magnetization which is nonzero. Much like the auxiliary field $f_{0}^{i}(x)$ acquires physical significance through a magnetic field $\vec{H}$, the quantity $h^{i}$ in (10.3) is related to a so-called anisotropy field $\vec{h}_{A}$. In any real magnetic system, there exist interactions whose description is beyond the reach of the isotropic Heisenberg Hamiltonian. One of these is magnetic anisotropy, which either originates from dipol-dipolinteractions between the spins or may be caused by the coupling of the electron orbits to the crystal fielde2 2 . In order to take these interactions into account on a microscopic level, one may introduce the artifice of an effective anisotropy field $\vec{h}_{A}$ into the microscopic Hamiltonian. For the antiferromagnet, which is then referred to as uniaxial, one obtains (see e.g.26. 43 )

$$
\mathcal{H}^{A F}=-J \sum_{n . n .} \vec{S}_{m} \cdot \vec{S}_{n}-\mu \sum_{n_{a} n_{b}}\left(S_{n_{a}}^{3}+S_{n_{b}}^{3}\right) \cdot H-\mu \sum_{n_{a} n_{b}}\left(S_{n_{a}}^{3}-S_{n_{b}}^{3}\right) \cdot h_{A} .
$$

In this model, which represents a special case of the Hamiltonian (10.2), the antiferromagnet is considered as composed of two sublattices $a$ and $b$, where $a$ - and $b$-spins are of equal 
magnitude. The arrangement is such that all nearest neighbors of an $a$-spin are $b$-spins and vice versa. In an idealized picture of the ground state, $a$-spins point up and $b$-spins point down. Note that, unlike for the external magnetic field before, we are now dealing with a hypothetical field, which changes its direction over atomic distances: $\vec{h}_{A}$ points along the positive $3^{\text {rd }}$ axis at $a$-sites, but along the negative $3^{\text {rd }}$ axis at $b$-sites.

As far as ferromagnets are concerned, magnetic anisotropies manifest themselves in a different manner in the microscopic description: again, they may be taken into account through an effective field, $\vec{H}_{A}$, which locally points in the same direction as every single spin vector; but here, they all point along one and the same direction. Accordingly, this field $\vec{H}_{A}$, which is also referred to as anisotropy field (see e.g.43-46), enters the microscopic Hamiltonian through the term $-\mu \sum_{n} \vec{S}_{n} \cdot \vec{H}_{A}$, i.e. it couples to the vector of the total spin, much like a magnetic field $\vec{H}$. Therefore, on the effective level, it is also to be incorporated into the quantities $f_{0}^{i}(x), f_{0}^{i}(x) \Leftrightarrow \mu H_{A}^{i}$, such that the qualitative effects of an external magnetic and an anisotropy field, respectively, are the same.

For the antiferromagnet, on the other hand, anisotropy field $\vec{h}_{A}$ and magnetic field $\vec{H}$ are not to be treated in analogous ways, since the quantity $\vec{h}_{A}$ does not couple to the vector of the total spin. It is instructive to discuss this novel interaction in our effective framework and confront the resulting modification of our previous findings with what is known in condensed matter physics.

Let us first examine the spin-wave dispersion relation. According to the preceding section, a magnetic field lifts the degeneracy of the two polarization states observed in an antiferromagnet, but does not provide the magnons with a "mass" - an anisotropy field $\vec{h}_{A}$, however, does the job. In the corresponding effective expansion,

$$
\Sigma_{s} \mu h_{A}^{i} U^{i}=\Sigma_{s} \mu h_{A}\left(1-\frac{1}{2} U^{a} U^{a}-\frac{1}{8} U^{a} U^{a} U^{b} U^{b}-\ldots\right),
$$

the term quadratic in the magnon variables leads to the relativistic scenario referred to in (9.6): in the presence of an anisotropy field, $\vec{h}_{A}=\left(0,0, h_{A}\right)$, as well as of an external magnetic field, $\vec{H}=(0,0, H)$, the dispersion law of antiferromagnetic spin waves takes the form 17

$$
\omega_{ \pm}=\sqrt{v^{2} \vec{k}^{2}+\Sigma_{s} \mu h_{A} / F_{1}^{2}} \pm \mu H
$$

Accordingly, the following relation holds,

$$
F_{1}^{2}\left(v^{2} M_{G B}\right)^{2}=\Sigma_{s} \mu h_{A}
$$

showing that the square of the "magnon mass" is proportional to the product of order parameter, $\Sigma_{s}$, and symmetry breaking parameter, $\mu h_{A}$. This formula may be viewed as the antiferromagnetic analog of the well-known Gell-Mann-Oakes-Renner relation encountered in QCD48,

$$
F_{\pi}^{2} M_{\pi}^{2}=|\langle 0|\bar{u} u| 0\rangle|\left(m_{u}+m_{d}\right) .
$$

The two equations are indeed in one-to-one correspondence: the square of the pion mass is determined by the product of the order parameter, the quark condensate $\langle 0|\bar{u} u| 0\rangle$, with the symmetry breaking parameter, the sum of the quark masses $m_{u}+m_{d}$. While the first factor 
is a measure of spontaneous symmetry breaking, the second one is a measure of explicit symmetry breaking.

Finally, let us consider how the scattering amplitude of antiferromagnetic spin waves is affected by the anisotropy field. From the expansion (10.5), we derive the following low energy theorem:

$$
\begin{gathered}
T^{A F}\left(\vec{h}_{A}\right)=\frac{1}{4 \sqrt{\omega_{1} \omega_{2} \omega_{3} \omega_{4}}} \frac{v^{4}}{F_{2}^{2}}\left\{\delta^{a b} \delta^{c d}\left(\frac{2}{v^{2}} \omega_{1} \omega_{2}-2 \vec{k}_{1} \cdot \vec{k}_{2}+\Sigma_{s} \mu h_{A} / F_{2}^{2}\right)\right. \\
+\delta^{a c} \delta^{b d}\left(2 \vec{k}_{1} \cdot \vec{k}_{3}-\frac{2}{v^{2}} \omega_{1} \omega_{3}+\Sigma_{s} \mu h_{A} / F_{2}^{2}\right) \\
\left.+\delta^{a d} \delta^{b c}\left(2 \vec{k}_{1} \cdot \vec{k}_{4}-\frac{2}{v^{2}} \omega_{1} \omega_{4}+\Sigma_{s} \mu h_{A} / F_{2}^{2}\right)\right\}
\end{gathered}
$$

where $\omega$ represents the modified dispersion relation, $\omega=\sqrt{v^{2} \vec{k}^{2}+\Sigma_{s} \mu h_{A} / F_{1}^{2}}$. Again, the formula (10.9) has its counterpart in QCD, if the pion-pion scattering amplitude is evaluated around nonzero quark mass.

In summary, the anisotropy field $\vec{h}_{A}$ is on the same footing as the quark masses - both quantities belong to those symmetry breaking parameters $m_{\alpha}$, which are not related to the generators of the symmetry group G. As we have pointed out in the analysis of symmetric underlying theories, the leading order effective Lagrangian of an antiferromagnet closely resembles the one describing QCD. Now, if the effective framework is extended to approximate symmetries, including an anisotropy field and quark masses, respectively, then the corresponding analogy in the low energy structure of the two theories is maintained.

\section{SYMMETRY BREAKING PARAMETERS}

We have to recall that the entire analysis in the last two sections, concerning approximate symmetries, relies on an essential assumption: the respective contributions, which explicitly break the symmetry of the underlying theory, are to be regarded as perturbations - the analysis in terms of effective fields is useful only, if the corresponding symmetry breaking parameters are sufficiently small. Let us now focus on this important requirement and discuss the various symmetry breaking parameters encountered so far from this point of view. We start with the anisotropy field.

Assuming that this field is weak, the predictions of the effective Lagrangian method, given in the previous section, can be trusted. For ferromagnets, as we have seen, magnetic anisotropies may be taken into account through an effective field $\vec{H}_{A}$, which is to be treated in the same way as a magnetic field $\vec{H}$ : the dispersion law of ferromagnetic spin waves experiences an overall shift linear in the perturbation,

$$
\omega=\gamma \vec{k}^{2}+\mu H_{A} .
$$

For antiferromagnets, where the anisotropy field, $\vec{h}_{A}$, does not couple to the generators of the group $\mathrm{O}(3)$, the perturbation shows up under a square root, the corresponding coefficient being proportional to the staggered magnetization,

$$
\omega=\sqrt{v^{2} \vec{k}^{2}+\Sigma_{s} \mu h_{A} / F_{1}^{2}}
$$


Hence, if the anisotropy fields, $\vec{H}_{A}$ and $\vec{h}_{A}$, respectively, are of the same order of magnitude and weak, the dispersion relation of antiferromagnetic spin waves exhibits a larger energy gap.

Indeed, this striking difference concerning the significance of anisotropy effects in ferroand antiferromagnets is well-known in condensed matter physics. In ferromagnets, these interactions only play a minor role, whereas in antiferromagnets they are much more pronounced: as a microscopic analysis, relying on some rough approximations, indicates (see e.g.49), the spin-wave spectrum of an antiferromagnet exhibits a characteristic energy gap,

$$
\Delta \omega=\mu \sqrt{h_{A}\left(2 h_{W}+h_{A}\right)}, \quad \vec{k} \rightarrow 0, \quad \vec{H} \rightarrow 0 .
$$

$h_{W}$ is the so-called Weiss field, which turns out to be very large compared to the anisotropy field, $h_{W} / h_{A} \approx 10^{3}$, such that the second term can be neglected. Accordingly, the above combination of anisotropy field and Weiss field, which does not show up in the analysis of ferromagnets, may lead to a substantial energy gap in the spin-wave spectrum of an antiferromagnet.

In particular, the formula for the energy gap (11.3) is consistent with the dispersion law (11.2): the contribution involving the Weiss field $h_{W}$ corresponds to the term involving the staggered magnetization $\Sigma_{s}$ - one identifies $h_{W} \Leftrightarrow \Sigma_{s} / 2 \mu F_{1}^{2}$. The other term appearing under the square root in (11.3), $\mu^{2} h_{A}^{2}$, is not reproduced by the effective theory: in our counting scheme, this expression corresponds to a contribution of subleading order and is thus beyond the reach of the leading order effective Lagrangian. Generally, our bookkeeping is based on a systematic counting of powers of momentum, such that the respective terms of a given order need not be correlated one-to-one with those obtained from a microscopic investigation of condensed matter.

In the case of very strong anisotropy effects, antiferromagnetic spin waves no longer follow the dispersion relation (11.2); rather, they obey a quadratic law $42, \omega=\alpha+\beta \vec{k}^{2}-$ the effective description no longer applies. Chiral perturbation theory, which is based on the assumption that the energy gap, associated with the Goldstone bosons, is small, now breaks down. The fact that the effective machinery only makes sense if the anisotropy field is weak, thus restricts the range of application of the method. However, as far as condensed matter systems are concerned, one has the freedom to chose appropriate objects of investigation one may easily find another antiferromagnetic body, displaying a weaker anisotropy field, and hence a smaller energy gap, such that the effective method now perfectly applies.

Note the difference with the description of the strong interaction: QCD is a universal theory - the symmetry breaking parameters, the quark masses, are fixed once and for all at their physical values. Up- and down-quarks are light, such that these quantities can be treated as perturbations. Next comes the strange-quark, whose mass is considerably larger, but nonetheless can be regarded as a perturbation, as well. The mass of the charmed quark, on the other hand, is much too large so as to be treated in an analogous manner. The three lightest quarks, however, may be viewed as perturbations of the symmetric Lagrangian of massless QCD, which is invariant under chiral transformations, $\mathrm{G}=\mathrm{SU}(3)_{R} \times \mathrm{SU}(3)_{L}$.

This so-called chiral limit, $m_{u}, m_{d}, m_{s} \rightarrow 0$, represents a purely theoretical abstraction - chiral symmetry is explicitly broken in nature. Likewise, a zero anisotropy field in an antiferromagnet is to be regarded as an idealized situation, too. As far as the third symmetry breaking parameter of interest, the external magnetic field, is concerned, the situation is 
different, because this quantity represents an external field. In a laboratory we can organize a world of our own, for we have the possibility to tune the strength of $\vec{H}$. In particular, the situation which is analogous to the fictitious chiral limit or a zero anisotropy field, can easily be realized: simply switch off the magnetic field. Then, at zero field strength, the ground state of a ferromagnet exhibits spontaneous magnetization - much like an antiferromagnet displays a nonzero staggered magnetization in the limit $\vec{h}_{A} \rightarrow 0$, or massless QCD exhibits a nonzero quark condensate.

Since the magnetic field can be varied continuously, the effective calculation is under control: as long as the field strength $|\vec{H}|$ is kept weak, the effective Lagrangian method is an efficient tool to investigate the low energy behavior of magnetic systems. Moreover, the fact that the magnetic field can be tuned, is a major advantage over QCD, where the quark masses are fixed: it provides us with a new way to accurately determine some of the low energy constants of the effective theory. Consider, for example, the magnetization of a ferromagnet and its variation with respect to temperature and magnetic field. In the effective expansion of this quantity, different low energy constants will show up. Their values may be unraveled by fitting the calculated curves to experimental data, which, furthermore, are already available in condensed matter physics. In particular, at higher orders of the low energy expansion, where the number of effective coupling constants turns out to be large, this procedure may be of considerable help.

\section{SUMMARY AND OUTLOOK}

The present work deals with the low energy analysis of nonrelativistic systems which exhibit collective magnetic behavior. The corresponding excitations near the ground state, the spin waves or magnons, are regarded as Goldstone bosons, resulting from a spontaneously broken internal symmetry, $\mathrm{O}(3) \rightarrow \mathrm{O}(2)$. Their properties may be analyzed in the framework of the effective Lagrangian method, which tackles the phenomenon of spontaneous symmetry breaking from a unified point of view. The method exploits the symmetry properties of the underlying theory and formulates the dynamics in terms of Goldstone fields.

At large wavelengths, the microscopic structure of condensed matter systems does not play a significant role: in the corresponding leading order effective Lagrangians, the specific properties of the system only manifest themselves in the numerical values of a few low energy couplings. Rather, our attention is devoted to the consequences of spontaneous symmetry breaking - it is the hidden symmetry which manifests itself at small momenta, dictating the explicit appearance of the respective low energy phenomenon.

If the ground state of the system fails to be Lorentz-invariant, charge densities may pick up nonzero vacuum expectation values. In the case of a ferromagnet, the spontaneous magnetization embodies this possibility, giving rise to a topological term in the effective Lagrangian, which is not invariant under the internal symmetry $\mathrm{O}(3)$. Ferromagnetic magnons are nonrelativistic particles, which possess only one polarization state and obey a quadratic dispersion relation. The low energy theorem concerning spin-wave scattering indeed displays a structure, which would not be permitted in the relativistic domain: the corresponding expressions for the scattering amplitude and total cross section violate Lorentz symmetry. The results obtained are in agreement with Dyson's pioneering microscopic analysis of a cubic ferromagnet within the Heisenberg model. 
The antiferromagnet, on the other hand, does not exhibit spontaneous magnetization, such that a topological term is absent in the effective Lagrangian. In contrast to the low energy excitations in a ferromagnet, antiferromagnetic magnons are relativistic particles, which follow a linear dispersion law and possess two polarization states. Much like in the relativistic domain, the effective Lagrangian is invariant with respect to the hidden symmetry $\mathrm{O}(3)$; moreover, the explicit expression for an antiferromagnet closely resembles the one referring to massless quantum chromodynamics (QCD). The T-matrix element, describing antiferromagnetic spin-wave scattering, unlike for the ferromagnet before, turns out to be Lorentz-invariant - its structure is analogous to that of the leading order pion-pion scattering amplitude in QCD, demonstrating the universal feature of the effective Lagrangian technique.

In either case, ferro- and antiferromagnetic spin-wave scattering, the calculation is readily done within the effective framework, to be contrasted with the microscopic approach, where the corresponding analysis turns out to be fairly involved. In this respect, the situation is analogous to that of quantum chromodynamics, where the complicated analysis of pionpion scattering by means of current algebra methods has been replaced by the effective Lagrangian technique. Unlike for the strong interaction, scattering processes in magnetic systems, unfortunately, are not the suitable tool to experimentally detect interactions among the Goldstone degrees of freedom.

The present work includes approximate symmetries and discusses the modification of the low energy structure imposed by explicit symmetry breaking. Two different perturbations of the isotropic Heisenberg model are considered: a constant external magnetic and a constant anisotropy field. The former quantity represents a rather special case, since this symmetry breaking parameter is coupled to the generators of the group $\mathrm{O}(3)$ - in the effective Lagrangian of a ferromagnet the magnetic field is associated with the spontaneous magnetization, while, for the antiferromagnet, it appears in the time component of a covariant derivative. The anisotropy field, on the other hand, which plays a significant role in connection with the antiferromagnet, belongs to the more general class of symmetry breaking parameters which are not coupled to the generators of the hidden symmetry. It leads to an additional term in the effective Lagrangian, which is proportional to the staggered magnetization.

The dispersion relations regarding spin waves in the presence of an external magnetic and an anisotropy field, respectively, are in agreement with the findings of condensed matter physics. Due to a magnetic field, ferromagnetic magnons experience an overall shift, while the degeneracy of the two polarization states of antiferromagnetic magnons is lifted. The anisotropy field provides antiferromagnetic magnons with a "mass", leading to a formula analogous to the Gell-Mann-Oakes-Renner relation in QCD.

Remarkably, to the order considered, the scattering process concerning ferromagnetic spin waves is not affected by a magnetic field. Also, the T-matrix element, describing the analogous interaction in an antiferromagnet, does not receive additional terms from the magnetic field. On the other hand, the anisotropy field modifies the low energy theorem concerning antiferromagnetic spin-wave scattering, leading to an additional contribution in the T-matrix element, which is on the same footing as the quark mass term in the pion-pion scattering amplitude.

The present work demonstrates that the leading order effective Lagrangians permit a con- 
cise and straightforward analysis of the low energy properties of ferro- and antiferromagnets, above all in connection with spin-wave scattering processes. The effective machinery may now be transferred to more complicated applications, such as the investigation of thermodynamic quantities. Indeed, the low temperature expansion for the partition function of an antiferromagnet has been calculated to three loops $\$ 0$, while the results concerning the temperature dependence of the spontaneous magnetization of a ferromagnet will be presented in a forthcoming paper 5 .

\section{ACKNOWLEDGMENTS}

I would like to thank H. Leutwyler for his patient assistance throughout this work and for his critical reading of the manuscript. Thanks also to G. Colangelo, P. Hasenfratz, S. Mallik, A. V. Manohar and D. Toublan for their help. I am greatly indebted to the Janggen-Pöhn-Stiftung for supporting my doctoral thesis. Likewise, support by Schweizerischer Nationalfonds is gratefully acknowledged.

\section{APPENDIX A: SPIN WAVES AS COLLECTIVE EXCITATIONS}

In this appendix, we develop a semiclassical picture of spin waves, which regards these low energy excitations as some kind of distortion of the microscopic spin structure $42,525$. Afterwards, we try to illuminate Goldstone's theorem in the present context. As a first step, we have to construct the microscopic representation of the one-magnon states.

Instead of the spin operators $S_{n}^{i}$, introduced in section $\mathbb{I}$, we take the following linear combinations thereof,

$$
S_{n}^{+}=S_{n}^{1}+i S_{n}^{2}, \quad S_{n}^{-}=S_{n}^{1}-i S_{n}^{2},
$$

and perform a discrete Fourier transformation:

$$
S^{ \pm}(\vec{k})=\sum_{n} \exp \left(i \vec{k} \vec{r}_{n}\right) S_{n}^{ \pm}
$$

Note that the operators $S^{ \pm}(\vec{k})$ refer to the reciprocal lattice.

Next, we apply these operators to the ground state of a ferromagnet. Since, in our convention, all of the spins point in the direction of the positive $3^{\text {rd }}$ axis, the spin-raising operator, $S^{+}(\vec{k})$, yields identically zero, $S^{+}(\vec{k})|0\rangle \equiv 0$. The spin-lowering operator, $S^{-}(\vec{k})$, however, leads to an eigenstate of the Heisenberg Hamiltonian,

$$
|\vec{k}\rangle=\frac{1}{\sqrt{2 S N}} S^{-}(\vec{k})|0\rangle, \quad\langle\vec{k} \mid \vec{k}\rangle=1 .
$$

$S$ is the spin quantum number and $N$ denotes the total number of lattice sites. Consider now the expectation value of the local operator $S_{n}^{3}$ in this one-magnon state (see e.g.42):

$$
\left\langle\vec{k}\left|S_{n}^{3}\right| \vec{k}\right\rangle=S-1 / N \text {. }
$$

This is quite a remarkable finding, since the right hand side is independent of the specific lattice site $n$ as well as of the wave vector $\vec{k}$. A spin deviation of one unit, relative to 
the ground state, is thus uniformly distributed over the whole lattice - per site $n$, the spin deviation from the totally ordered state equals $1 / N$. In this picture, a spin wave corresponds to some sort of collective excitation moving through the lattice, characterized by a wave vector $\vec{k}$. Since the total spin deviation amounts to one unit, these quasiparticles are bosons - so far so good.

In accordance with the semiclassical vector model, each localized spin which takes part in such a collective mode precesses around the $3^{r d}$ axis. The corresponding opening angle is such that the projection of a particular spin vector on the $3^{r d}$ axis is given by $S-1 / N$. Moreover, there is a constant phase difference between any two adjacent spins of the collective mode, depending on the magnitude of the wave vector $\vec{k}$ - in particular, at large wavelengths, $|\vec{k}| \rightarrow 0$, all the spins precess in phase.

Now, in order to get an intuitive understanding of Goldstone's theorem in the present context, let us briefly discuss a simple model of a ferromagnet司. Consider a linear chain of $N$ spin- $\frac{1}{2}$ vectors, bent around into a ring, such that the first and the $(N+1)^{t h}$ spin are identical. Suppose that there is an interaction between adjacent spins, tending to align them parallel. The ground state, in which all the spins point in the same direction, is degenerate. From the manifold of these $N+1$ lowest lying states let us chose, for definiteness, the one with maximal spin projection $N / 2$ on the $z$-axis. Next, construct a one-magnon state $|k\rangle$,

$$
\begin{gathered}
|k\rangle=\frac{1}{\sqrt{N}} \sum_{n} e^{i k n}|n\rangle, \quad k=\frac{2 \pi}{N} r, \quad r=1,2, \ldots, \\
|n\rangle=\frac{1}{2}\left(\sigma_{n, x}-i \sigma_{n, y}\right)|0\rangle,
\end{gathered}
$$

where the quantities $\sigma_{n}$ represent Pauli matrices. Now, for $k=0$, the one-magnon state $|k\rangle$ is energetically degenerate with $|0\rangle$ - these two states differ, however, with respect to the projection of their total spin on the third axis: for $|k=0\rangle$, one obtains $N / 2-1$. In particular, this state may be regarded as another of the possible ground states, whose "spontaneous magnetization" points in a direction different from the third axis or, equivalently, with a different value of the third component of the spin vector along the same direction. These two configurations are thus related by a symmetry transformation: with a suitable rotation, the ground state, $|0\rangle$, may be transformed into $|k=0\rangle$.

Reasoning by analogy, we may transfer these statements to a three-dimensional ferromagnet. In particular, such a rotation of the system as a whole would not require any energy: since the spin structure of the ground state is not distorted while rotating the rigid spin lattice, there are no restoring forces. As a consequence, there exists at least one form of elementary excitation, the one corresponding to $\vec{k}=0$, which gives a rotation of the entire system in spin space, and which must have zero frequency, $\omega=0$ 逐. These $"(\vec{k}=0 / \omega=0)$ excitations" above the ground state, however, are just other ground states and the real question rather is, whether there is some sort of excitation with no energy gap in the limit $\vec{k} \rightarrow 023$. Under one additional condition, the absence of long-range forces, there will indeed exist a whole branch in the spectrum of elementary excitations, whose frequency continuously tends to zero, $\omega \rightarrow 0$, in the limit $\vec{k} \rightarrow 0$ - here we recognize the nonrelativistic version of Goldstone's theorem.

Imagine an external field, causing a slight distortion of the ordered spin structure, such that the direction of the magnetization slowly varies in space, periodically over some characteristic length $\lambda$. If the external field is switched off, the system begins to oscillate with 
some characteristic frequency or frequencies. Goldstone's theorem then deals with the question whether or not such collective modes have an energy gap as the characteristic length in the original distortion of the order parameter tends to infinity. Since the summation in the Heisenberg exchange Hamiltonian merely extends over nearest neighbors, by definition, no long-range forces are present in this model. Therefore, Goldstone excitations do occur.

\section{APPENDIX B: SCATTERING AMPLITUDE AND CROSS SECTION}

As is well-known from relativistic quantum mechanics, the cross section referring to elastic two-particle scattering is given by

$$
d \sigma=\frac{\left|T^{R}\right|^{2}}{4 \sqrt{\left(k_{1} \cdot k_{2}\right)^{2}-m_{1}^{2} m_{2}^{2}}}(2 \pi)^{4} \delta^{4}\left(k_{1}+k_{2}-k_{3}-k_{4}\right) \frac{d^{3} k_{3}}{(2 \pi)^{3} 2 \omega_{3}} \frac{d^{3} k_{4}}{(2 \pi)^{3} 2 \omega_{4}},
$$

with

$$
\delta^{4}\left(k_{1}+k_{2}-k_{3}-k_{4}\right)=\delta\left(\omega_{1}+\omega_{2}-\omega_{3}-\omega_{4}\right) \delta^{3}\left(\vec{k}_{1}+\vec{k}_{2}-\vec{k}_{3}-\vec{k}_{4}\right) .
$$

For collinear collisions, the Lorentz-invariant square root may be expressed by the relative velocity $|\vec{v}|$ of the ingoing particles 57.58 ,

$$
\omega_{1} \omega_{2}|\vec{v}|=\sqrt{\left(k_{1} \cdot k_{2}\right)^{2}-m_{1}^{2} m_{2}^{2}}, \quad|\vec{v}|=\left|\vec{v}_{1}-\vec{v}_{2}\right| .
$$

Proceeding this way, we do not specialize to the dispersion law of massive relativistic particles. Note that a relativistic normalization of the one-particle states has been used,

$$
\left\langle\vec{k} \mid \vec{k}^{\prime}\right\rangle=(2 \pi)^{3} 2 \omega \delta^{3}\left(\vec{k}-\vec{k}^{\prime}\right) \Longleftrightarrow \int \frac{d^{3} k}{(2 \pi)^{3} 2 \omega}|\vec{k}\rangle\langle\vec{k}| g
$$

Likewise, in Born approximation, the quantity $T^{R}$,

$$
T^{R}=\left\langle\vec{k}_{3} \vec{k}_{4}\left|\mathcal{L}_{\text {int }}\right| \vec{k}_{1} \vec{k}_{2}\right\rangle^{R}
$$

is the $T$-matrix element, evaluated in this specific normalization.

Since our analysis of ferro- and antiferromagnets is based on a nonrelativistic normalization of the one-particle states,

$$
\left\langle\vec{k} \mid \vec{k}^{\prime}\right\rangle=(2 \pi)^{3} \delta^{3}\left(\vec{k}-\vec{k}^{\prime}\right) \quad \Longleftrightarrow \int \frac{d^{3} k}{(2 \pi)^{3}}|\vec{k}\rangle\langle\vec{k}|,
$$

the expression (B.1) has to be modified accordingly: instead of normalizing to $2 \omega$ particles in a given volume $V$, we shall normalize to one particle per volume $V$. Hence, the formula for the scattering cross section now reads:

$$
d \sigma=\frac{\left|T^{N R}\right|^{2}}{|\vec{v}|}(2 \pi)^{4} \delta^{4}\left(k_{1}+k_{2}-k_{3}-k_{4}\right) \frac{d^{3} k_{3}}{(2 \pi)^{3}} \frac{d^{3} k_{4}}{(2 \pi)^{3}} .
$$


The quantity $T^{N R}$ then represents the $T$-matrix element

$$
T^{N R}=\left\langle\vec{k}_{3} \vec{k}_{4}\left|\mathcal{L}_{\text {int }}\right| \vec{k}_{1} \vec{k}_{2}\right\rangle^{N R},
$$

where the one-particle states as well as the field operators, appearing in the interaction Lagrangian, are normalized nonrelativistically.

We are now going to derive the explicit expression for the differential cross section, assuming that the interacting particles obey a quadratic dispersion relation, $\omega=\gamma \vec{k}^{2}$. In connection with condensed matter, it is appropriate to consider the specific frame of reference, which is given by the solid body at rest. Accordingly, the initial particle configuration is to be characterized by two arbitrary wave vectors, $\vec{k}_{1}$ and $\vec{k}_{2}$. It is convenient to choose ingoing and outgoing momenta as follows,

$$
\begin{array}{ll}
\vec{k}_{1}=\frac{1}{2}(\vec{K}+\vec{q}), & \vec{k}_{2}=\frac{1}{2}(\vec{K}-\vec{q}), \\
\vec{k}_{3}=\frac{1}{2}\left(\vec{K}+\vec{q}^{\prime}\right), & \vec{k}_{4}=\frac{1}{2}\left(\vec{K}-\vec{q}^{\prime}\right),
\end{array}
$$

where the vectors $\vec{K}$ and $\vec{q}$,

$$
\vec{K}=\vec{k}_{1}+\vec{k}_{2}, \quad \vec{q}=\vec{k}_{1}-\vec{k}_{2}
$$

represent total and relative momentum, respectively. In the case of quadratic kinematics, conservation of energy and momentum leads to $|\vec{q}|=\left|\vec{q}^{\prime}\right|$. The scattering angle $\vartheta$ is chosen as the angle between the direction of $\vec{q}^{\prime}$ relative to that of $\vec{q}$. In these coordinates, the evaluation of the phase space integral is readily done, resulting in

$$
\int \delta^{4}\left(k_{1}+k_{2}-k_{3}-k_{4}\right) d^{3} k_{3} d^{3} k_{4} \rightarrow \frac{1}{8 \gamma}|\vec{q}| d \Omega .
$$

With the relative velocity of the ingoing particles,

$$
|\vec{v}|=2 \gamma\left|\vec{k}_{1}-\vec{k}_{2}\right|=2 \gamma|\vec{q}|
$$

the expression for the differential cross section then amounts to

$$
\frac{d \sigma}{d \Omega}=\frac{1}{128 \pi^{2} \gamma^{2}}\left|T^{N R}\right|^{2}
$$

Note that a factor of one half has been included in the above formula, considering the fact that the interacting particles are identical. 


\section{REFERENCES}

* On leave from Institute for Theoretical Physics, University of Bern, Sidlerstrasse 5, CH3012 Bern, Switzerland.

${ }^{1}$ S. Weinberg, Phys. Rev. Lett. 18, 188, 507 (1967); Phys. Rev. 166, 1568 (1968);

R. Dashen, Phys. Rev. 183, 1245 (1969);

R. Dashen and M. Weinstein, Phys. Rev. 183, 1261 (1969).

${ }^{2}$ S. Coleman, J. Wess and B. Zumino, Phys. Rev. 177, 2239 (1969);

C. G. Callan, S. Coleman, J. Wess and B. Zumino, Phys. Rev. 177, 2247 (1969).

${ }^{3}$ L.-F. Li and H. Pagels, Phys. Rev. Lett. 26, 1204 (1971);

H. Pagels, Phys. Rep. C 16, 219 (1975).

${ }^{4}$ S. Weinberg, Physica A 96, 327 (1979).

${ }^{5}$ J. Gasser and H. Leutwyler, Ann. Phys. (N.Y.) 158, 142 (1984); Nucl. Phys. B 250, 465 (1985).

${ }^{6}$ P. Hasenfratz and H. Leutwyler, Nucl. Phys. B 343, 241 (1990).

${ }^{7}$ P. Hasenfratz and F. Niedermayer, Phys. Lett. B 268, 231 (1991).

${ }^{8}$ P. Hasenfratz and F. Niedermayer, Z. Phys. B 92, 91 (1993).

${ }^{9}$ H. Leutwyler, Phys. Rev. D 49, 3033 (1994).

${ }^{10}$ H. Leutwyler, Helv. Phys. Acta 70, 275 (1997).

${ }^{11}$ R. M. Roman and J. Soto, Effective Field Theory Approach to Ferromagnets and Antiferromagnets in Crystalline Solids, preprint Univ. of Barcelona UB-ECM-PF 97/23, condmat/9709298.

${ }^{12}$ C. P. Burgess, Goldstone and Pseudogoldstone Bosons in Nuclear, Particle and Condensed Matter Physics, hep-th/9808176.

${ }^{13}$ H. Leutwyler, Ann. Phys. 235, 165 (1994).

${ }^{14}$ E. Fradkin, Field Theories of Condensed Matter Systems, Frontiers in Physics Vol. 82 (Addison-Wesley, 1991).

${ }^{15}$ F. J. Dyson, Phys. Rev. 102, 1217 (1956).

${ }^{16} \mathrm{H}$. Leutwyler, in Hadron Physics 94 - Topics on the Structure and Interaction of Hadronic Systems, edited by V. E. Herscovitz, C. A. Z. Vasconcellos and E. Ferreira (World Scientific, 1995), p. 1.

${ }^{17}$ A recent analysis, considering the manifestation of the crystal point group $\overline{3} m$ at nextto-leading order in the effective Lagrangian for ferro- and antiferromagnets, can be found in 11 .

${ }^{18}$ We identify the zeroth component of the coordinate vector with time: $x^{0}=t$ (no factor of c).

${ }^{19}$ J. Goldstone, Nuovo Cim. 19, 154 (1961);

J. Goldstone, A. Salam and S. Weinberg, Phys. Rev. 127, 965 (1962).

${ }^{20}$ S. Coleman, Erice Lectures 1973, in Laws of Hadronic Matter (Academic Press, London and New York, 1975); reprinted in S. Coleman, Aspects of Symmetry (Cambridge Univ. Press, 1985).

${ }^{21}$ G. S. Guralnik, C. R. Hagen and T. W. B. Kibble, in Advances in Particle Physics, edited by R. L. Cool and R. E. Marshak (Wiley, New York, 1968), Vol. 2, p. 567.

${ }^{22}$ The phase of the Goldstone-boson states $\left|\pi^{a}(\vec{k})\right\rangle$ may be chosen such that the constant $F$ is real and positive.

${ }^{23}$ R. V. Lange, Phys. Rev. Lett. 14, 3 (1965); Phys. Rev. 146, 301 (1966). 
${ }^{24}$ H. B. Nielsen and S. Chadha, Nucl. Phys. B 105, 445 (1976).

25 The zeroth component of the momentum vector is identified with the energy, $k^{0}=\omega$ (no factor of c); moreover, $k x \equiv \omega t-\vec{k} \vec{x}$.

${ }^{26}$ C. Kittel, Quantum Theory of Solids (Wiley, New York, 1987).

${ }^{27}$ L. D. Landau and E. M. Lifshitz, Statistical Physics, edited by E. M. Lifshitz and L. P. Pitajewski, Course of Theoretical Physics Vol. 9 (Pergamon, London, 1981), Part 2.

${ }^{28}$ S. Randjbar-Daemi, A. Salam and J. Strathdee, Phys. Rev. B 48, 3190 (1993).

${ }^{29}$ E. D'Hoker and S. Weinberg, Phys. Rev. D 50, 6050 (1994).

${ }^{30}$ For a more detailed account and. particularly, for a derivation of the results given here, the reader may consult reference $\mathrm{\theta}$.

31 J. Bijnens, G. Colangelo, G. Ecker, J. Gasser and M. E. Sainio, Phys. Lett. B 374, 210 (1996).

${ }^{32}$ More precisely, QCD with an exact chiral symmetry, $\mathrm{G}=\mathrm{SU}(2)_{R} \times \mathrm{SU}(2)_{L}$ broken down to $\mathrm{H}=\mathrm{SU}(2)_{V}$.

${ }^{33}$ Since the effective representation of the current (8.2) is proportional to $\varepsilon_{i j k}$, and the corresponding transition matrix element (5.8) involves a Kronecker delta, the tensor $\varepsilon_{a b}$ will appear either in the definition of the field operators or the one-magnon-states. We choose the latter possibility.

${ }^{34}$ F. Keffer and R. Loudon, J. Appl. Phys. (Suppl.) 32, 2 (1961).

${ }^{35}$ T. Oguchi, Phys. Rev. 117, 117 (1960).

${ }^{36}$ D. Beeman and P. Pincus, Phys. Rev. 166, 359 (1968).

${ }^{37}$ A. Brooks Harris, Phys. Rev. 183, 486 (1969).

${ }^{38}$ V. N. Kashcheev, Soviet Phys. - Solid State 4, 556 (1962).

${ }^{39}$ Note that a relativistic normalization of the one-pion states has been used, $\left\langle\pi^{a}(\vec{k}) \mid \pi^{b}\left(\vec{k}^{\prime}\right)\right\rangle=(2 \pi)^{3} \delta^{a b} 2 k^{0} \delta^{3}\left(\vec{k}-\vec{k}^{\prime}\right) . k^{0}$ is the time component of the momentum four vector, $k^{\mu}=(\omega / c, \vec{k})$.

40 The whole analysis still refers to leading order of the effective expansion - the low energy theorems to be derived below receive corrections due to higher order terms, i.e. terms of higher orders in the momenta than $\vec{k}^{2}$.

${ }^{41}$ In order to avoid confusion with signs, the magnetic moment $\mu$ is taken positive: the spin vectors and the spontaneous magnetization, respectively, thus point along the same direction as the external magnetic field. This convention will be maintained throughout the paper.

${ }^{42}$ W. Nolting, Quantentheorie des Magnetismus (Teubner, Stuttgart, 1986), Band 2.

43 J. van Kranendonk and J. H. van Vleck, Rev. Mod. Phys. 30, 1 (1958).

${ }^{44}$ F. Keffer, Spin Waves, in Encyclopedia of Physics - Ferromagnetism, edited by S. Flügge and H. P. J. Wijn (Springer, Berlin, 1966), Vol. 18-2, p. 1.

${ }^{45}$ S. W. Tjablikow, Quantentheoretische Methoden des Magnetismus (Teubner, Leipzig, 1968)

${ }^{46}$ A. Herpin, Théorie du Magnétisme (Presses Universitaires de France, Paris, 1968).

${ }^{47}$ Note that, for antiferromagnets, the magnetic field $\vec{H}$ occurs on the same footing as the wave vector $\vec{k}, f_{0}^{i}(x) \propto|\vec{k}|$, whereas the anisotropy field $\vec{h}_{A}$ counts as quantity of order two, $m_{\alpha}(x) \propto \vec{k}^{2}$.

${ }^{48}$ M. Gell-Mann, R. J. Oakes and B. Renner, Phys. Rev. 175, 2195 (1968).

${ }^{49}$ D. Wagner, Introduction to the Theory of Magnetism (Pergamon, Oxford, 1972). 
${ }^{50}$ C. P. Hofmann, Effective Analysis of the $O(N)$ Antiferromagnet: Low Temperature Expansion of the Order Parameter, preprint Univ. Bern BUTP-97/15, hep-ph/9706418, submitted to Phys. Rev. B.

${ }^{51}$ C. P. Hofmann, in preparation.

${ }^{52}$ G. Heller and H. A. Kramers, Proc. Roy. Acad. Amsterdam 37, 378 (1934).

${ }^{53}$ F. Keffer, H. Kaplan and Y. Yafet, Amer. J. Phys. 21, 250 (1953).

${ }^{54}$ C. Kittel, Introduction to Solid State Physics (Wiley, New York, 1986).

${ }^{55}$ W. Heisenberg, Einführung in die einheitliche Feldtheorie der Elementarteilchen (Hirzel, Stuttgart, 1967).

${ }^{56}$ P. W. Anderson, Concepts in Solids (Benjamin, New York, 1963).

${ }^{57}$ W. Greiner and J. Reinhardt, Quantenelektrodynamik, Theoretische Physik Band 7 (Harri Deutsch, Thun, 1984).

${ }^{58}$ I. J. R. Aitchison and A. J. G. Hey, Gauge Theories in Particle Physics (A. Hilger, Bristol, 1982). 